Research Report

Proposed journal section: Cognitive Neuroscience / Clinical and Translational Neuroscience

\title{
ATTENTIONAL MODULATION OF NEURAL DYNAMICS IN TACTILE PERCEPTION OF COMPLEX REGIONAL PAIN SYNDROME PATIENTS
}

Serena Defina ${ }^{1}$, Maria Niedernhuber ${ }^{1}$, Nicholas Shenker ${ }^{2}$, Christopher Brown ${ }^{2,3}$ and Tristan A. Bekinschtein $^{1}$

${ }^{1}$ Consciousness and Cognition Lab, Department of Psychology, University of Cambridge, Cambridge, United Kingdom.

${ }^{2}$ Department of Rheumatology, Addenbrooke's Hospital, Cambridge University Hospitals NHS Foundation Trust, Cambridge, United Kingdom.

${ }^{3}$ Department of Psychological Sciences, University of Liverpool, Liverpool, United Kingdom.

Corresponding authors: NS [ nicholas.shenker@addenbrookes.nhs.uk ]; TAB [ tb419@,cam.ac.uk ]

Running title: Altered tactile processing in CRPS

Keywords: CRPS; Perceptual disturbances; EEG; Multivariate Analysis; Hierarchical Predictive Coding. 
Total nr of pages: 36; Figures: 6; Tables: 2; Words: 5392 (whole manuscript), 221 (Abstract).

\title{
Acknowledgements
}

This research was funded by BJA/RCoA (Project Grant ID WKR0-2018-0060 to TAB) and Cambridge Arthritis Research Endeavour (CARE to NS). The current analysis was conducted thanks to Erasmus + for traineeship funds.

\begin{abstract}
Body perceptual disturbances are an increasingly acknowledged set of symptoms and possible clinical markers of Complex Regional Pain Syndrome (CRPS), but the neurophysiological and neurocognitive changes that underlie them are still far from being clear. We adopted a novel multivariate and neurodynamical approach to the analysis of EEG modulations evoked by touch, to highlight differences between patients and healthy controls, between affected and unaffected side of the body, and between "passive" (i.e. no task demands and equiprobable digit stimulation) and "active" tactile processing (i.e. where a digit discrimination task was administered and spatial probability manipulated). Contrary to our expectations we found no support for early differences in neural processing between CRPS and healthy participants, however, there was increased decodability in the CRPS group compared to healthy volunteers between 280 and $320 \mathrm{~ms}$ after stimulus onset. This group difference seemed to be driven by the affected rather than the unaffected side and was enhanced by attentional demands. These results found support in the exploratory analysis of neural representation dynamics and behavioural modelling, highlighting the need for single participant analyses. Although several limitations impacted the robustness and generalizability of our comparisons, the proposed novel analytical approach yielded promising insights (as well as possible biomarkers based on neural dynamics) into the relatively unexplored alterations of tactile decisionmaking and attentional control mechanisms in chronic CRPS.
\end{abstract}




\section{Introduction}

Body perception is a fundamental ingredient of human conscious experience (Ehrsson et al., 2004; Lenggenhager et al., 2007; Blanke et al., 2015) and a strikingly relevant aspect of such experience, especially when we are in pain. A growing number of neuroimaging studies and neurocognitive models are starting to uncover the complex, multilevel, system of interactions between cortical and subcortical brain areas and afferent somatosensory information, that underpin body perception during pain (e.g., (Woo et al., 2017; Mouraux \& Iannetti, 2018)). As it is often the case, peculiar distortions of body perception that emerge in certain chronic pain conditions, have sparked precious insights into the complexities of this important brain function. Complex Regional Pain Syndrome (CRPS) is a debilitating and poorly understood condition, in which disproportionate pain and a variable combination of other symptoms follows an apparently minor injury to one of the limbs (Bruehl, 2015; Birklein et al., 2018). Although this eerie "unilateral" pain condition has raised fascination and perplexity for decades, its etiology and treatment remain elusive, also because of the disarming heterogeneity of its clinical presentation (Marinus et al., 2011). In recent years, the observation of a disparate and peculiar set of disturbances in body perception (Lewis \& Schweinhardt, 2012) (e.g., finger misperception) (Förderreuther et al., 2004; Kuttikat et al., 2017) in some of these patients has tilted the investigation of the pathophysiology of the disease towards possible (structural and functional) cortical changes in somatosensory brain regions (Swart et al., 2009). The initial idea, developed in analogy to other pain conditions such as phantom limb or chronic back pain (Flor et al., 1995; Flor et al., 1997; Bray \& Moseley, 2011), was that misperception symptoms could result from CRPS-induced maladaptive plasticity (i.e. cortical reorganization) in the contralateral primary sensory cortex (S1) (Maihöfner et al., 2004; Pleger et al., 2004). However, the few neuroimaging studies conducted so far have led to contradicting results, spanning from a similar representation of affected and unaffected sides of the body, to an enlarged (rather than shrunk) representation of the affected limb onto S1 (Di Pietro et al., 2013; Di Pietro et al., 2015; van Velzen et al., 2016; Mancini et al., 2019; Pfannmöller et al., 2019). However, as recently pointed out by some authors (Kuttikat et 
al., 2018; Brown et al., 2020), most work on CRPS-related body misperception (mirroring the larger branch of body perception research) has traditionally focused on early, more "physiological" components of somatosensory processing (i.e. < 50 ms; e.g., (Pleger et al., 2004; Lenz et al., 2011)), relying on experimental architectures that are more concerned with stimulation precision rather than its ecological validity. Yet, later-latency, more "cognitive" factors such as attentional modulation and perceptual decision making have long been known to modify somatosensation (Mima et al., 1998; Franz et al., 2015), including that of pain (Bantick et al., 2002; Chan et al., 2012; Clauwaert et al., 2020) and, the growing evidence of executive dysfunction in CRPS (Apkarian et al., 2004; Geha et al., 2008; Lee et al., 2015), led some to suggest that the contradictory patterns of neural representation alterations emerging from CRPS literature could be partially explained by differential cognitive and attentional engagement elicited different paradigms.

Besides these conceptual limitations, studies on CRPS-related functional plasticity are guilty of a number of further methodological issues, namely, for instance, the lack of adequate spatial resolution and accuracy of the currently available source reconstruction methods (Maihöfner et al., 2003; Kuttikat et al., 2016) and the limited sensitivity offered by average-based signal analysis techniques such as ERPs. Indeed, classical (univariate) ERP analyses by definition mask the variability across subjects in order to let similarities emerge (Luck, 2005), but individual differences in patterns of activation do exist and, especially for more heterogeneous groups of people, like CRPS patients definitely are, group contrasts are easily overwhelmed by the amount of inter-individual noise. Multivariate pattern analysis (MVPA; or "brain decoding") is gaining exponential popularity in the world of cognitive (and clinical) neuroscience, thanks to the promise of increased sensitivity and reduced reliance on "spatially variant" confounds such us, e.g., anatomical differences in the folding pattern of the cortex (King \& Dehaene, 2014; Grootswagers et al., 2017). MVPA offers additional advantages when investigating the temporo-spatial dynamics of pain processing (Rosa \& Seymour, 2014) and it has successfully been used to decode pain experience and sensitivity in heathy volunteers (Schulz et al., 2011; Tu et al., 2014; Lancaster et al., 2017). However, applications of decoding 
techniques to EEG signals (that are conveniently portable, cheap, and offer excellent temporal precision) have never been used in combination with attentional demand manipulations to study (painful) somatosensation in healthy humans, nor in any clinical setting, at least to our knowledge. Hence, we aimed at investigating the influence of attentional modulation on CRPS-related perceptual alterations, by using multivariate pattern classification of early, mid-latency and late EEG activity elicited by tactile digit stimulation and recognition. We exploited MVPA methodological advantages to better reflect and leverage individual patterns of "neural information" and highlight differences between CRPS patients and healthy volunteers (HV) and between the affected and unaffected side in individual patients. We did so not only during "rest" (i.e. passive stimulation) but also under increased "cognitive" demands (i.e. active condition, where spatial probability was manipulated, and a digit discrimination task was administered). As per our hypotheses (https://osf.io/rmhsb), we expected:

H.1. A main effect of group (CRPS $<\mathrm{HV})$ on mean performance in distinguishing the five fingers from each other (i.e. all-vs-all decoding).

H.2. An interaction of group by side (left and right) on all-vs-all finger classifier accuracy, in the direction of smaller difference between hands in the HV group.

H.3. A main effect of side (affected $<$ unaffected) on classifier performance for brain activity elicited by stimulation of the thumb (D1) vs. the little finger (D5) (i.e. 1-vs-5 decoding), across conditions (passive and active stimulation), in any of the CRPS patients.

H.4. An interaction of condition by side-affected on 1-vs-5 decoding accuracy, such that, in the active but not in the passive condition the performance in the affected hand will be comparable to that of the healthy (unaffected) hand.

Despite their mostly descriptive and exploratory nature, we believe our results shed important insight into the possible neurophysiological and neurocognitive basis of tactile misperceptions reported in CRPS, opening a window for a conceptually and methodologically new approach to this field of investigation. 


\section{Materials and methods}

\section{Participants}

Our sample included 13 patients (11 females, mean(range) age $=46.8(30-63)$ years) who were diagnosed with unilateral upper or lower limb CRPS according to modified Budapest Research Criteria (Harden et al., 2007) and 13 age and sex matched healthy volunteers (11 females, mean(range) age $=45.0(28-63)$ years). All participants (patients and healthy volunteers) were righthanded. Exclusion criteria were: previous or current diagnosis of peripheral neuropathy, stroke, transient ischemic attack, multiple sclerosis, malignancy or seizure disorder.

\section{Ethical standards}

All participants gave written and informed consent to take part in the study and were remunerated for their time. The study was approved by the Research Ethics Committee of East of England-South Cambridge (REC Ref No: 12/EE/0305) and it conforms with the World Medical Association Declaration of Helsinki (2013). All possible measures were taken to minimize pain or discomfort in all participants, with a special attention to the CRPS group. All patients were advised to report immediately if the sensation was uncomfortable or painful and the fingertips were also checked after each session for any redness of the skin. After every block of sensory stimulation, a break of at least 10 seconds (in the passive condition) or a few minutes (in the active condition) was offered to improve participants' comfort.

\section{Study design}

Two custom-made handboxes (one for each hand) were calibrated to deliver soft touch stimuli with the same force to the fingertips of each digit. The experiment, as depicted in Figure 1, consisted of 4 blocks of passive stimulation (i.e. passive condition), followed by 8 blocks of finger recognition task (i.e. active condition). In the passive condition, participants were instructed sit relaxed and keep their eyes closed head still as much as possible. In each passive block, 50 touches were delivered in random order to each finger of one hand. Left and right hand were alternated between blocks, for a total of 
500 trials per hand (100 for each finger). In the active condition, participants placed one hand on the handbox, with their eyes still closed and a relaxed but still posture; again, hands were alternated between blocks. This time, participants were asked to respond to the stimulation by saying out loud the number corresponding to the stimulated finger $(i . e$. thumb $=1$, index finger $=2$, middle finger $=$ 3 , ring finger $=4$ and little finger $=5$ ) and a bottom-up manipulation of predictability was implemented, with outer fingers (D1 and D5, i.e. "expected") being touched on $75 \%$ of all trials, whereas the probability of stimulation for central fingers (D2, D3 or D4, i.e., "rare" / "oddball" fingers) was $25 \%$. The answer was recorded manually by the experimenter thereby triggering the next stimulus. Each active block consisted of 80 trials, for a total of 320 stimulations for each hand (120 to each thumb, 120 to each little finger, and 27 each for the remaining three rare fingers).

\section{EEG acquisition and preprocessing}

During both conditions, 128-channel high-density EEG data were collected using the Net Amps 300 amplifier (Electrical Geodesics Inc., Oregon, USA), at the sampling frequency of $250 \mathrm{~Hz}$. Raw data were pre-processed in MATLAB using the EEGLAB toolbox (Delorme \& Makeig, 2004) and inhouse scripts. Channels on the neck, cheeks and forehead were removed, leaving 92 retained channels for further analysis. EEG data were filtered between 0.5 and $30 \mathrm{~Hz}$ and epoched between $-200 \mathrm{~ms}$ and $800 \mathrm{~ms}$ relative to stimulation onset. Artefacts originating from eye blinks, eye or muscle movements and electrical interference were identified and removed by means of visual inspection and independent component analysis (ICA). After the interpolation of rejected channels, data were rereferenced to the average and baseline-corrected relative to a $200 \mathrm{~ms}$ interval before the stimulation. For each participant, single-subject ERP plots were examined to ensure data quality.

\section{MVPA and Statistical Analysis}

Multivariate Pattern Analysis (MVPA) on EEG data was implemented using MATLAB-based ADAM toolbox (Fahrenfort et al., 2018). We employed a Linear Discriminant Analysis (LDA) algorithm to perform decoding (Grootswagers et al., 2017) and a 10-fold stratified cross-validation 
method was implemented to prevent overfitting. Hence, we ran the classification 10 times; each time the LDA model was fit (or "trained") on $9 / 10$ of the total number of trials, leaving the remaining $10 \%$ for testing. The procedure was then repeated for each time point in the trial. Imbalances within and between classes resulting from noisy trial rejection and/or experimental manipulations were corrected by means of undersampling. Brain decoding was performed first at a "super-subject" level for both (active and passive) conditions. A super-subject was built by "stacking" together all trials from all healthy volunteers, regardless of who performed them. The resulting "super-block" was then analysed as a single subject dataset, following the procedure described above. Super-subject classifier performance was calculated for each finger (against all others) individually, averaged across the resulting five time-courses and used to select the time window(s) of interest for subsequent analysis, according to cluster-based permutation testing with significance level of 0.05 . For the "all-vs-all" analysis, a multi-class LDA was trained to assign the input brain pattern to one of five finger classes (D1, D2, D3, D4 and D5). To quantify the decoding performance, Classification Accuracy (CA) was calculated per class, by averaging across the 10 folds (at each time-point), then averaged across classes and compared to the random classification rate (RCR), defined as the reciprocal of the number of classes (i.e. 20\%). For the "1-vs-5" analysis, a linear classifier was fit to the trials corresponding to stimulation of the thumb (D1) and little finger (D5) in both conditions and hands of the CRPS group. Classifier performance was measured using Area Under the Curve (AUC), a nonparametric criterion-free measure of generalization (Grootswagers et al., 2017). To asses statistical significance (hypothesis H.1 and H.2), the selected time-windows of "all-vs-all" decoding output for the two groups and sides were compared using a Linear Mixed Model with "side" (affected vs unaffected), "group" (CRPS vs HV) and their interaction estimated as fixed effects, whereas the intercept values were allowed to vary across subjects (i.e. random coefficient). Because the difference between active and passive activations became evident in later stages of processing, we examined it by adding a main effect of condition among the fixed coefficients of the model. Finally, to test hypothesis H.3 and H.4, each patients' data was compared in a two-way repeated-measures ANOVA featuring side (affected 
and unaffected) and condition (passive and active) as main factors. Statistical analysis was implemented in R and Jamovi (Şahin \& Aybek, 2019) and a p-value <.05 was considered statistically significant.

\section{Exploratory analysis}

To explore whether the trends detected by our analysis would mirror any differences in the actual behavioral choices of our participants in the finger recognition task, we calculated $d^{\prime}$ (d-prime) and bias (c) scores by opposing "expected" (D1 and D5) and "rare" (D2-D4) digits. Originally borrowed from Signal Detection Theory (SDT), $d$ ' and $c$ are meant to decompose response behavior in order to capture the individual's sensitivity (i.e., the ability to distinguish the two alternative sensory signals) and his/her "decision criterion", providing an indication of any "preference" for one or the other answer (Wickens, 2002; Macmillan \& Creelman, 2004; Bang \& Rahnev, 2017). In our case, $c$ is expected to reflect the effect of expectation induced by digit probability manipulation. In other words, an "optimal" decision criterion would be slightly displaced towards negative values, as responses "1" and "5" are more probably correct and therefore "preferred" over "2", "3" or "4". The same model used for group-level analysis of classifier output was adopted to predict both these scores (i.e., a Linear Mixed Model with side, group and their interaction estimated as fixed effects, whereas the intercept of each subject as a random coefficient).

We also assessed the temporal stability/mutability of these finger mental representations in the two groups, conditions, and sides of the body, by applying Temporal Generalization Analysis (TGA), a decoding technique that, instead of training and testing a classifier on data from a single timepoint at the time as we described above, tests each classifier trained on time $t$ using all other timepoints in the trial (King \& Dehaene, 2014). If the same pattern of information (e.g., the representation of a finger) recurs at multiple time points, that pattern is deemed stable. If the generalizability is low despite above-chance decoding, then the neural information that supports such decoding is likely not as stable but rather it evolves dynamically across time. 
All the scripts used for the neural and behavioral analyses and for the displaying of results can be found at https://github.com/SereDef/CRPS-decoding-behavioral-modeling.

\section{Results}

\section{Healthy "super-subject” results}

We defined the temporal regions of interest with the mean "super-subject" classifier (illustrated in Figure 2). This analysis showed a performance reliably better than chance within three temporal clusters: between 28 and $56 \mathrm{~ms}$ (peak: $40 \mathrm{~ms}$ ) and between 84 and $128 \mathrm{~ms}$ (peak: $112 \mathrm{~ms}$ ) after stimulus onset in the passive condition; and between 364 and $500 \mathrm{~ms}$ (peak: $480 \mathrm{~ms}$ ) after stimulus onset in the active condition. The same temporal patterns appeared when all-vs-all and a 1-vs-5 decoder were trained and tested on the same healthy "super-subject" data. We therefore selected an “early" (28-56 ms), "mid" (84-128 ms) and "late" (364-500 ms) time windows for statistical testing. We cautiously decided to include a fourth "mid-late" time window, extending from 180 to $320 \mathrm{~ms}$ after stimulus presentation, as a large peak in decoding performance seems to be present in the active condition (Figure 2D), potentially relevant for cognitive processing.

\section{Group-level results}

The performance (i.e. CA) time-courses of the all-vs-all decoder is represented in Figure 3. For each subject, we selected four temporal clusters of CA values (early, mid, mid-late and late time windows) and averaged CA across time within those windows. Within the same windows the peak (highest performance value) and its latency were also registered and analysed for exploration purposes. All results including F-values for each comparison, their associated p-values and effect sizes are reported in Table 1.

We found little evidence supporting our first hypothesis - i.e. lower mean decoding performance in the CRPS group compared to controls - in any of the four time-windows. An effect in the predicted direction was present in the early time-window only, but not to a significant extent $\left(F_{1,24}=2.96\right.$, $\left.P=0.098, \eta^{2} p=0.054\right)$. Notably however, a reliable effect of group, in the opposite direction compared 
to our predictions (i.e., CRPS $>$ HV) emerged in the mid-late time-window of $180-320 \mathrm{~ms}\left(F_{1,24}=7.37\right.$, $\left.P=0.012, \eta^{2} p=0.095\right)$, further corroborated by exploratory analysis of peak performance $\left(F_{1,24}=8.66\right.$, $\left.P=0.007, \eta^{2} p=0.095\right)$. There was no support for our second hypothesis - H.2 - , stating a larger difference between hands in the CRPS group compared to HV. A small but reliable interaction of group by side emerged in the early time window $\left(F_{1,24}=5.39, P=0.029, \eta^{2} p=0.041\right)$, CA was slightly higher (0.01 point increase) in the left hand compared to the right one for healthy volunteers, and slightly lower (0.02 point decrease) on the side affected by CRPS compared to the unaffected one in the patient group.

An expected result not formally stated a priori was the effect of attention. The decoders trained on the active condition performing reliably better than the passive ones in the two later time windows $\left(F_{1,51}=75.40, P<0.001, \eta^{2} p=0.387 ; F_{1,75}=94.92, P<0.001, \eta^{2} p=0.429\right)$.

\section{Individual-level results}

The subject-wise differences between the two sides (affected and unaffected) in each condition and timeframe are summarised in Figure 4, and all statistical results (including F-values, p-values and effect sizes) are fully reported in Table 2.

There was no reliable effect for our third hypothesis of better performance in the unaffected side compared to the painful one. We found an effect of side in the predicted direction for H.3 in 3 of 13 patients $\left(F_{1,9}=7.80, P=0.021, \eta^{2} p=0.464 ; F_{1,9}=5.72, P=0.040, \eta^{2} p=0.388 ; \quad F_{1,9}=7.77, P=0.021\right.$, $\left.\eta^{2} p=0.463\right)$. None of the 13 participants affected by CRPS showed interactions as we hypothesized in H.4, however, in 6/13 of the patients' results showed the "shape" in the predicted direction. An effect of side leaning in the opposite direction with respect to our prediction (i.e., the affected side performing better than the healthy one) was present in two other patients $\left(F_{1,9}=12.21, P=0.007\right.$, $\eta^{2} p=0.576 ; F_{1,9}=5.54, P=0.043, \eta^{2} p=0.381$ ), one of which also showed an interaction of side by condition $\left(F_{1,9}=21.24, P=0.001, \eta^{2} p=0.702\right)$, but in the opposite direction to our hypothesis H.4. 


\section{Exploratory results}

The Temporal Generalization matrices resulting from our exploratory TGA within the two groups, sides and conditions are represented in Figure 5. The first considerable difference that stands out (by contrasting Figures 5A, C and E to Figures 5B, D and F) is how information processing changes radically when task demands are introduced in the active condition. While during passive stimulation (5A-C-E) a more diagonal-shaped decoding performance indicates neural patterns that generalize over a brief, transient time period only, higher levels of temporal generalition emerge during the active condition (5B-D-F), suggesting cortical signals (or mental representations) that are "mantained" more stably actross time. Secondly, when we focus on the active processing differences between sides of the body, i.e. unaffected (5B) and affected (5D) by the CRPS, is noticeable how, while the unaffected side shows a generalization pattern that is comparable to that of healthy volunteers $(5 \mathrm{~F})$, with a more widespread decodability expanding to timepoints that even preced sitimulus onset, the affected side TGA seems to yield a narrower and "delayed" spread of finger neural representation.

With respect to the behavioral variables (represented in Figure 6), a significant main effect of group $\left(F_{1,24}=5.83, P=0.024, \eta^{2} p=0.158\right)$ and side $\left(F_{1,24}=12.63, P=0.002, \eta^{2} p=0.065\right)$ emerged on $d^{\prime}$ 'scores. Sensitivity scores were 0.48 SDs higher on average on the unaffected / dominant hand compared to the contralateral one, and 0.70 units lower in CRPS patients compared to the HV group. Even though the interaction effect was not reliable $\left(F_{1,24}=0.54, P=0.470, \eta^{2} p=0.003\right)$, the difference in performance between the two hands (i.e., affected $<$ unaffected) was higher in the CRPS group $\left(F_{1,24}=9.20\right.$, $P=0.006)$, compared to that of healthy volunteers $\left(F_{1,24}=3.97, P=0.058\right)$. With respect to the bias $(c)$ score, none of the fixed effect included in the model did yield significant results (Group effect: $F_{1,24}=3.27, P=0.083 \eta^{2} p=0.078$; Side: $F_{1,24}=2.42, P=0.133, \eta^{2} p=0.020$; Group $*$ Side: $F_{1,24}=0.82$, $P=0.373, \eta^{2} p=0.006$ ). However, as evident from Figure 6 , the main difference between the two 
groups' behavioral prerfomance was really in the accentuated spread or variability of such measures across the patients' group compared to healthy controls.

\section{Discussion}

We did not find consistent support for a worse tactile decoding performance in the CRPS group compared to healthy controls, nor for a greater decodability gap between hands in these patients in any of our time windows of interest (H.1 and H.2). In addition, three patients showed a significantly worse classification performance on the affected (compared to the unaffected) side of the body (H.3), but none of our patients presented the expected reduction of decodability gap between hands during the active task, compared to the passive stimulation (H.4). Nonetheless, the adoption of time series MVPA has proven useful novel tool for the investigation of tactile processing differences induced by CRPS. It is encouraging to notice, for instance, how the "super-subject" decoding time-course did yield similar "peaks" to those often reported in in classical somatosensory ERP research: the earliest cortical components emerging between $20-80 \mathrm{~ms}$, followed by a N1-like wave (commonly observed around 80-120 ms) (Luck, 2005) can be appreciated in both conditions (Figure 2). These results lend further support to the thesis that CRPS does not show changes in the fine grained cortical somatotopies (Mancini et al., 2019). These two early modulations are meant to mirror the more "physiological" aspects of sensory processing, tracking afferent inputs from peripheral nerves into primary cortical areas (S1; <50ms (Lueders et al., 1983; Wood et al., 1988)), and from there to secondary processing stages (S2; $>80 \mathrm{~ms}$ ), building up to the (yet unconscious) detection of sensory stimuli (Schubert et al., 2006; Schubert et al., 2008; Garrido et al., 2009; Chennu et al., 2013). Interestingly, when an attentional modulation is introduced by task demands in the active condition (Figure 2B and 2D) two further and wider performance peaks emerge between 250 and $500 \mathrm{~ms}$ (Figure 2B), strongly resembling the notorious P3 (a and b) modulation (Polich, 2007). 
A disease-induced "temporal dragging" of sensory information processing

This latter "P3-like" task-induced attentional modulation constitutes a statistically robust effect that emerged from our analysis and we argue that these later-latency, more "cognitive" stages of processing, ultimately may be the ones that enshrine the key to perceptual differences induced by chronic CRPS. Indeed, the amount of neural information detected by our classifiers was abnormally enhanced (rather the reduced) in the CRPS group between 180 and $320 \mathrm{~ms}$ after the touch has been delivered (Figure 3B). This result, in line with the prior analysis of this same dataset (Kuttikat et al., 2018) as well as other recent theoretical accounts (Brown et al., 2020), suggests that later ("P2 / P3like") components of processing, supporting higher cognitive functions such as attentional allocation and perceptual decision-making, assume greater importance in CRPS patients compared to controls. This arguably "compensatory" shift in the temporal distribution of tactile information processing, i.e., a relative dampening of early performance peaks followed by heavier reliance on later processing, is evident not only in the active condition (Figure 3B), but also to a lesser extent during passive stimulation. Indeed, by looking at the decoding output in Figure 3A one can notice how two lower performance peaks in CRPS (vs. HV) round $\sim 50$ and $\sim 100 \mathrm{~ms}$ are followed by a "dragging" of decoding sustained over 200-250 ms into the trial, when HV decoder is already at chance. This observation also matches previous evidence of greater amplitude of mid-late ERP components in CRPS patients (Kuttikat et al., 2018) and potentially signals further processing or delays of sensory sensation leaking into central cognitive control or awareness. The passive "dragging effect" emerges again from TGA (Figure 5A and C vs. Figure 5E), stressing again how in CRPS patients compared to HVs, a shade of relevant finger information does persist beyond basic sensory processing stages, despite no instructions are currently deeming that information useful to fulfil any higher-level task.

\section{An affected-side-specific "temporal shift" into later processing stages}

Although the effect of side as we expected it (affected $<$ unaffected) did not reach a robust statistical support neither at the group nor at an individual level, when zooming into the decodability timecourses corresponding to the affected and unaffected sides of the body (Figure 3E and 3F) some 
interesting patterns emerge. In the passive condition for instance (Figure 3E), one could notice how the above mentioned "dragging" of performance around 200-250 ms in the CRPS group (Figure 3A) seems to be mostly driven by the affected side, rather than by the unaffected counterpart. This affected-side-specific docking of neural information onto a time-frame that is thought to underlie more cognitive stages of processing (rather than early perceptual/sensory), stresses again the idea that more advanced and abstract aspects of perception may be altered by the disease, especially on the side of the body that is directly affected by it. Similarly, in the active condition (Figure 3F), one can appreciate how the CRPS-related dampening of the first peak in Figure 3B ( $\sim 50 \mathrm{~ms}$, supposedly underlying poorer primary somatosensory processing, and mirrored in the passive condition in Figure $3 \mathrm{~A}$ and $3 \mathrm{E}$ ) seems to originate again more from the affected rather than the unaffected side of the body. Interestingly, the exploratory analysis of the temporal dynamics of processing in the affected and unaffected sides of CRPS patients (Figure 5B and 5D) helped highlighting a considerable temporal shift in finger representation that seems specific to the affected side, but could not emerge by simple diagonal (i.e., transient) decodability (Figure 3F). More research is needed to clarify these side-specific dynamics of perceptual information processing in CRPS, but here we highlight a potentially crucial role of attentional demands in such distinction (Bultitude et al., 2017).

\section{Intersubject variability: an issue and a resource}

Besides the underappreciated role of later stages of processing in CRPS-related somatosensory misperceptions, the second most consistent result emerging from our analysis is that of a substantial inter-subject variability in the CRPS group. We snapshot this variability in Figure 4, by representing the mean decoding difference between the two hands (i.e., "decodability imbalance") in each participant, condition, and time-window. There, while healthy volunteers (Figure 4B) are more consistent in showing a slightly better performance on the dominant hand across conditions and timeframes (with higher imbalances early in the trial quickly resolved after 80ms), CRPS patients show less overlap between conditions and a less consistent pattern across the group and across time. Interestingly, behavioral results point again to a more variable picture in the CRPS group compared 
to HVs. Even though patients did show lower sensitivity $\left(d^{\prime}\right)$ scores than controls on average (Figure $6 \mathrm{~A})$, most of the variability in $d$ ' scores was due to interindividual variability rather than any of the fixed effects in the model, and by inspecting Figure 6C, one can appreciate how the spread of $d$ ' values is relatively larger in the CRPS group compared to controls. Similarly, while the expected negative criterion shift (Bang \& Rahnev, 2017) is consistently present across healthy participants, CRPS patients' decisional process seems much less consistent (Figure 6B and 6D) and overall less finely tuned to statistical optimality (Brown et al., 2020). In light of this issue, future analyses oriented towards leveraging rather than silencing these inter-individual differences, can greatly contribute to our understanding of misperception phenomena in CRPS.

\section{Theoretical interpretation: Hierarchical Predictive Coding}

The journey of CRPS through possible theoretical explanations and framings has been a troubled one (Feliu \& Edwards, 2010; Borchers \& Gershwin, 2014; Popkirov et al., 2019). Most recently, some authors began to appeal to the idea that, in order to understand the complex and subtle perceptual phenomena accompanying the disease, an articulated and thorough model of human perception needs to be taken into account (Kuttikat et al., 2016; Kuttikat et al., 2018; Popkirov et al., 2019; Brown et al., 2020). If we model the human brain (including that of CRPS patients) under a framework that regards its mechanisms as a complex, hierarchical - possibly Bayesian - structure, we can then assume perception is simultaneously and dynamically influenced not only by afferent inputs and forward sensory processing steps but also by higher-level knowledge. In a nutshell, this long-standing perspective, often referred to as Hierarchical Predictive Coding (HPC) models (Friston, 2018), proposes that information accumulated and distilled from prior experiences is constantly used to predict or "explain away" new sensory inputs; if the new experience is completely conforming to such expectations, no further processing is needed, but when something surprising (i.e., not matching the existing prediction) is present in the incoming sensory stream, a residual prediction error is generated. These errors propagate forward in the system and are used to update the expectations and improve future predictions, i.e., learning (Feldman \& Friston, 2010). Two aspects of HPC theories 
are relevant when trying to understand tactile misperceptions in CRPS. One hypothesis is that ambiguity in sensory input (resulting for instance from sensory nerve pathology in some forms of CRPS) biases perception towards expectations, implying an abnormally strong influence of cognitive predictions on perception. Some evidence pointing in this direction is that lower frequency bands (e.g., delta and theta ranges) of brain activity, thought to underlie top-down predictions, show greater spectral power across frontal and somatosensory cortices in CRPS patients compared to controls (Walton et al., 2010). The same idea also finds moderate support in our group-level decoding output (Figure 3A and 3B), where an initial lesser decodability in the CRPS (vs. HV) in the first ( 50ms) peaks, is inverted in later time frames (after $\sim 180 \mathrm{~ms}$; our mid-late time-window). Zooming into the active identification task, a heavier reliance on expectancy-related information could be a plausible explanation of the group gain in classifier performance (Figure 3B) as well as the affected-side specific delay in establishing a stable finger mental representation to support digit identification (Figure 5D). This "shift" of processing (described above) could be interpreted as a "compensatory" mechanism, that leverages the intact top-down neural structure to a newly lesioned and inefficient afferent stream, but, an internal model of the world that is less efficient to the incoming information ultimately becomes too rigid and bound to miss the optimal interpretation of such sensory input. Indeed, our behavioral exploration showed a much less uniform estimated displacement of decision criterion ( $c$; Figure 6B and 6D) in the patient group, and this was especially true for the affected side of the body. CRPS patients' perceptual decisions were less tuned towards the optimal value with respect to the real probability of stimulation (Bang \& Rahnev, 2017) and this idea echoes with other similar but independent analyses of CRPS-related changes in perceptual predictions (Brown et al., 2020). Altogether, the picture that seems to emerge is that of a lack of balance between top-down and bottom-up influences on perception, which brings us to the other key player in this complex Bayesian game of perception: attention. In the HPC framework, attention (or "salience") serves as a balancecontrol system, enhancing or dampening the influence of the other model components (bottom-up or top-down signals) according to the respective "precision" weights, i.e., degree of certainty (Chennu 
et al., 2013; Moran et al., 2013). This could be relevant to CRPS research, for two reasons. First, pain (especially chronic pain) is known to drain attentional resources from other parallel processes (Dick et al., 2003) thereby impairing identification performance on the affected, but also potentially on the unaffected side. Second, a lack of attention (or precision) on the affected side could prevail in patients with more severe cognitive neglect-like symptoms (Kuttikat et al., 2016; Wittayer et al., 2018), resulting in worse top-down/bottom-up balancing on the affected rather than the unaffected side. This potentially concurring antagonist mechanisms could explain why, despite an affected-side specific delay in processing was evident from TGA outcomes (Figure 5B-D) the difference between sides in terms of behavioral (Figure 6) and diagonal decoding performances (Figure 3F) was less clear and inconsistent across the patient group, as emerged from the individual-level statistical results as well.

\section{Conclusion}

Altogether, the puzzle of symptoms and neural information patters characterizing this enigmatic condition seems to point to a difficulty with the body mental representation (Kuttikat et al., 2016; Halicka et al., 2020), rather than a mere deficit in peripheral transmission (Yvon et al., 2018) or modifications of cortical somatotopies (Mancini et al, 2019). Expectancy-related and attentional aspects of sensory processing deserve more investigation as contributing mechanisms to CRPS perceptual disturbances, a combination of novel decoding techniques and computational models such as HPC may be a useful toolset for understanding the cognitive and pathophysiological changes related to them. More research is needed to clarify side-specific and general dynamics of perceptual information processing in CRPS, ideally supported by principled experimental design and guided by the ultimate goal to leverage individual differences to boost understanding, prevention and treatment of the disorder.

\section{Conflict of Interest Statement}

The authors have no conflicts of interest to report. 


\section{Author Contributions}

TB and NS were responsible for the conceptualisation and preparation of study protocol. Anoop Kuttikat recruited the subjects, administered the tests and collected all EEG data. CB provided the data and, together with $\mathrm{TB}$, NS, and $\mathrm{MN}$ offered vital feedback on analysis planning and interpretation of the results. SD and TB prepared the preregistration and SD analysed the data. SD, MN, NS, CB and TB wrote the paper based on SD Master Thesis.

\section{Data Accessibility Statement}

All data supporting the findings of this study are available upon request to $\mathrm{CB}$. The data are not publicly available as they contain sensitive information that could compromise the privacy of research participants.

\section{Abbreviations}

CA - Classification Accuracy; CRPS - Complex Regional Pain Syndrome; EEG Electroencephalography; ERP - Evoked Related Potential; HPC - Hierarchical Predictive Coding; HV - Healthy Volunteers; MVPA - Multivariate Pattern Analysis; RCR - Random Classification Rate; S1 - Primary Somatosensory Cortex; S2 - Secondary Somatosensory Cortex; TGA - Temporal Generalization Analysis. 


\section{Tables}

Table 1. Summary of all group-level statistical tests. A total of twelve models were estimated (three models for each of the four time-windows). The conditional $\mathrm{R}^{2}$ values of each model are indicated between brackets. F-scores, degrees of freedom (df), $p$-values and partial $\eta^{2}$ are reported for each comparison. Significant results (under the uncorrected threshold of 0.05) are highlighted in bold.

\begin{tabular}{|c|c|c|c|c|}
\hline Factors & $\mathrm{F}$ & df & $\mathrm{p}$ & $\eta^{2} \mathrm{p}$ \\
\hline \multicolumn{5}{|c|}{ Early Mean Accuracy $\left(\right.$ model $\left.\mathrm{R}^{2}=0.41\right)$} \\
\hline Diagnosis & 2.959 & $1 / 24$ & 0.098 & 0.055 \\
\hline Side & 0.010 & $1 / 24$ & 0.920 & 0.000 \\
\hline Condition & 0.836 & $1 / 51$ & 0.365 & 0.006 \\
\hline Diagnosis $*$ Side & 5.385 & $1 / 24$ & 0.029 & 0.041 \\
\hline \multicolumn{5}{|c|}{ Early Peak $\left(\right.$ model $\left.\mathrm{R}^{2}=0.35\right)$} \\
\hline Diagnosis & 2.032 & $1 / 24$ & 0.167 & 0.037 \\
\hline Side & 0.000 & $1 / 24$ & 0.979 & 0.000 \\
\hline Condition & 0.054 & $1 / 51$ & 0.818 & 0.000 \\
\hline Diagnosis $*$ Side & 1.391 & $1 / 24$ & 0.250 & 0.011 \\
\hline \multicolumn{5}{|c|}{ Early Peak Latency $\left(\right.$ model $\left.\mathrm{R}^{2}=0.35\right)$} \\
\hline Diagnosis & 0.549 & $1 / 24$ & 0.466 & 0.008 \\
\hline Side & 8.603 & $1 / 24$ & 0.007 & 0.095 \\
\hline Condition & 0.432 & $1 / 51$ & 0.514 & 0.003 \\
\hline Diagnosis $*$ Side & 0.366 & $1 / 24$ & 0.551 & 0.004 \\
\hline
\end{tabular}

\begin{tabular}{|c|c|c|c|c|}
\hline Factors & $\mathrm{F}$ & df & $\mathrm{p}$ & $\eta^{2} \mathrm{p}$ \\
\hline \multicolumn{5}{|c|}{ Mid Mean Accuracy $\left(\right.$ model $\left.\mathrm{R}^{2}=0.30\right)$} \\
\hline Diagnosis & 0.000 & $1 / 24$ & 0.979 & 0.000 \\
\hline Side & 1.000 & $1 / 71.2$ & 0.321 & 0.007 \\
\hline Condition & 1.878 & $1 / 75$ & 0.175 & 0.014 \\
\hline Diagnosis $*$ Side & 0.039 & $1 / 71.2$ & 0.843 & 0.000 \\
\hline \multicolumn{5}{|c|}{ Mid Peak $\left(\right.$ model $\left.\mathrm{R}^{2}=0.41\right)$} \\
\hline Diagnosis & 0.200 & $1 / 24$ & 0.659 & 0.004 \\
\hline Side & 0.373 & $1 / 24$ & 0.547 & 0.002 \\
\hline Condition & 0.448 & $1 / 51$ & 0.506 & 0.003 \\
\hline Diagnosis $*$ Side & 0.415 & $1 / 24$ & 0.526 & 0.003 \\
\hline \multicolumn{5}{|c|}{ Mid Peak Latency $\left(\right.$ model $\left.\mathrm{R}^{2}=0.26\right)$} \\
\hline Diagnosis & 9.134 & $1 / 24$ & 0.006 & 0.095 \\
\hline Side & 4.557 & $1 / 75$ & 0.036 & 0.042 \\
\hline Condition & 2.410 & $1 / 75$ & 0.125 & 0.023 \\
\hline Diagnosis $*$ Side & 0.942 & $1 / 75$ & 0.335 & 0.009 \\
\hline
\end{tabular}

\begin{tabular}{|c|c|c|c|c|}
\hline Factors & $\mathrm{F}$ & df & $\mathrm{p}$ & $\eta^{2} \mathrm{p}$ \\
\hline \multicolumn{5}{|c|}{ Mid-late Mean Accuracy (model $\left.\mathrm{R}^{2}=0.52\right)$} \\
\hline Diagnosis & 7.374 & $1 / 24$ & 0.012 & 0.095 \\
\hline Side & 2.183 & $1 / 24$ & 0.153 & 0.020 \\
\hline Condition & 75.399 & $1 / 51$ & $<.001$ & 0.387 \\
\hline Diagnosis $*$ Side & 0.335 & $1 / 24$ & 0.568 & 0.003 \\
\hline \multicolumn{5}{|c|}{ Mid-late Peak $\left(\right.$ model $\left.\mathrm{R}^{2}=0.54\right)$} \\
\hline Diagnosis & 8.664 & $1 / 24$ & 0.007 & 0.095 \\
\hline Side & 4.050 & $1 / 75$ & 0.048 & $\mathbf{0 . 0 3 7}$ \\
\hline Condition & 84.342 & $1 / 75$ & $<.001$ & 0.444 \\
\hline Diagnosis $*$ Side & 0.001 & $1 / 75$ & 0.974 & 0.000 \\
\hline \multicolumn{5}{|c|}{ Mid-late Peak Latency $\left(\right.$ model $\left.\mathrm{R}^{2}=0.09\right)$} \\
\hline Diagnosis & 0.183 & $1 / 47.2$ & 0.671 & 0.002 \\
\hline Side & 0.419 & $1 / 49.6$ & 0.520 & 0.004 \\
\hline Condition & 4.997 & $1 / 75$ & 0.028 & $\mathbf{0 . 0 4 7}$ \\
\hline Diagnosis $*$ Side & 3.098 & $1 / 49.6$ & 0.085 & 0.031 \\
\hline
\end{tabular}

\begin{tabular}{|c|c|c|c|c|}
\hline Factors & $\mathrm{F}$ & $\mathrm{df}$ & $\mathrm{p}$ & $\eta^{2} \mathrm{p}$ \\
\hline \multicolumn{5}{|c|}{ Late Mean Accuracy $\left(\right.$ model $\left.\mathrm{R}^{2}=0.55\right)$} \\
\hline Diagnosis & 0.120 & $1 / 24$ & 0.733 & 0.002 \\
\hline Side & 0.513 & $1 / 75$ & 0.476 & 0.004 \\
\hline Condition & 94.917 & $1 / 75$ & $<.001$ & 0.429 \\
\hline Diagnosis $*$ Side & 0.138 & $1 / 75$ & 0.711 & 0.001 \\
\hline \multicolumn{5}{|c|}{ Late Peak $\left(\right.$ model $\left.\mathrm{R}^{2}=0.55\right)$} \\
\hline Diagnosis & 0.029 & $1 / 24$ & 0.865 & 0.000 \\
\hline Side & 1.453 & $1 / 69.3$ & 0.232 & 0.013 \\
\hline Condition & 109.48 & $1 / 75$ & $<.001$ & 0.497 \\
\hline Diagnosis $*$ Side & 0.040 & $1 / 69.3$ & 0.842 & 0.000 \\
\hline \multicolumn{5}{|c|}{ Late Peak Latency $\left(\right.$ model $\left.\mathrm{R}^{2}=0.18\right)$} \\
\hline Diagnosis & 2.587 & $1 / 31.9$ & 0.118 & 0.026 \\
\hline Side & 1.680 & $1 / 25.4$ & 0.207 & 0.020 \\
\hline Condition & 2.391 & $1 / 75$ & 0.126 & 0.021 \\
\hline Diagnosis $*$ Side & 0.067 & $1 / 25.4$ & 0.798 & 0.001 \\
\hline
\end{tabular}


Table 2. Summary of all individual-level statistical tests. Each model was estimated on the single patient's data and indicated by the patient id. F-scores, degrees of freedom (df), p-values and partial $\eta^{2}$ are reported for each comparison. Significant results (according to the uncorrected threshold of $0.05)$ are highlighted in bold.

\begin{tabular}{|c|c|c|c|c|}
\hline Factors & $\mathrm{F}$ & $\mathrm{df}$ & $\mathrm{p}$ & $\eta^{2} \mathrm{p}$ \\
\hline \multicolumn{5}{|c|}{ P04 } \\
\hline Condition & 2.30 & $1 / 9$ & 0.163 & 0.204 \\
\hline Side & 2.29 & $1 / 9$ & 0.164 & 0.203 \\
\hline Condition $*$ Side & 3.48 & $1 / 9$ & 0.095 & 0.279 \\
\hline \multicolumn{5}{|c|}{$P 07$} \\
\hline Condition & 2.67 & $1 / 9$ & 0.137 & 0.229 \\
\hline Side & 7.80 & $1 / 9$ & 0.021 & 0.464 \\
\hline Condition $*$ Side & 3.60 & $1 / 9$ & 0.090 & 0.286 \\
\hline \multicolumn{5}{|c|}{ P08 } \\
\hline Condition & 21.01 & $1 / 9$ & 0.001 & 0.700 \\
\hline Side & 12.21 & $1 / 9$ & 0.007 & 0.576 \\
\hline Condition $*$ Side & 21.24 & $1 / 9$ & 0.001 & 0.702 \\
\hline \multicolumn{5}{|c|}{$P 10$} \\
\hline Condition & 6.45 & $1 / 9$ & 0.032 & 0.417 \\
\hline Side & 0.02 & $1 / 9$ & 0.896 & 0.002 \\
\hline Condition 註 Side & 3.05 & $1 / 9$ & 0.115 & 0.253 \\
\hline \multicolumn{5}{|c|}{$P 12$} \\
\hline Condition & 3.52 & $1 / 9$ & 0.093 & 0.281 \\
\hline Side & 1.01 & $1 / 9$ & 0.341 & 0.101 \\
\hline Condition $*$ Side & 0.61 & $1 / 9$ & 0.457 & 0.063 \\
\hline \multicolumn{5}{|c|}{$P 13$} \\
\hline Condition & 0.71 & $1 / 9$ & 0.420 & 0.073 \\
\hline Side & 2.39 & $1 / 9$ & 0.157 & 0.209 \\
\hline Condition $*$ Side & 2.08 & $1 / 9$ & 0.183 & 0.187 \\
\hline \multicolumn{5}{|c|}{$P 14$} \\
\hline Condition & 7.55 & $1 / 9$ & 0.023 & 0.456 \\
\hline Side & 6.25 & $1 / 9$ & 0.034 & 0.410 \\
\hline Condition $*$ Side & 4.65 & $1 / 9$ & 0.059 & 0.341 \\
\hline
\end{tabular}

\begin{tabular}{|c|c|c|c|c|}
\hline Factors & $\mathrm{F}$ & df & $\mathrm{p}$ & $\eta^{2} p$ \\
\hline \multicolumn{5}{|c|}{ P15 } \\
\hline Condition & 5.63 & $1 / 9$ & 0.042 & 0.385 \\
\hline Side & 0.32 & $1 / 9$ & 0.584 & 0.035 \\
\hline Condition $*$ Side & 2.15 & $1 / 9$ & 0.176 & 0.193 \\
\hline \multicolumn{5}{|c|}{ P16 } \\
\hline Condition & 0.42 & $1 / 9$ & 0.533 & 0.045 \\
\hline Side & 5.72 & $1 / 9$ & 0.040 & 0.388 \\
\hline Condition $*$ Side & 3.93 & $1 / 9$ & 0.079 & 0.304 \\
\hline \multicolumn{5}{|c|}{ P17 } \\
\hline Condition & 2.058 & $1 / 9$ & 0.185 & 0.186 \\
\hline Side & 5.541 & $1 / 9$ & 0.043 & 0.381 \\
\hline Condition $*$ Side & 0.174 & $1 / 9$ & 0.686 & 0.019 \\
\hline \multicolumn{5}{|c|}{ P18 } \\
\hline Condition & 6.60 & $1 / 9$ & 0.030 & 0.423 \\
\hline Side & 0.37 & $1 / 9$ & 0.556 & 0.040 \\
\hline Condition $*$ Side & 0.81 & $1 / 9$ & 0.392 & 0.082 \\
\hline \multicolumn{5}{|c|}{ P19 } \\
\hline Condition & 0.24 & $1 / 9$ & 0.637 & 0.026 \\
\hline Side & 0.11 & $1 / 9$ & 0.745 & 0.012 \\
\hline Condition $*$ Side & 0.09 & $1 / 9$ & 0.765 & 0.010 \\
\hline \multicolumn{5}{|c|}{$P 20$} \\
\hline Condition & 1.39 & $1 / 9$ & 0.269 & 0.134 \\
\hline Side & 7.77 & $1 / 9$ & 0.021 & 0.463 \\
\hline Condition $*$ Side & 0.48 & $1 / 9$ & 0.508 & 0.050 \\
\hline
\end{tabular}




\section{Figures}

Figure 1 - Experimental Design

\section{A. PASSIVE CONDITION}

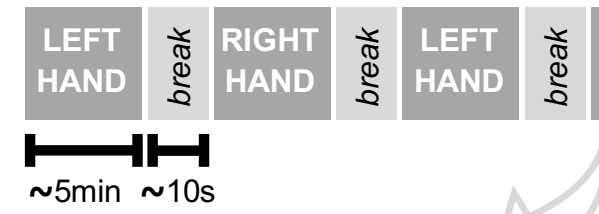

50 trials

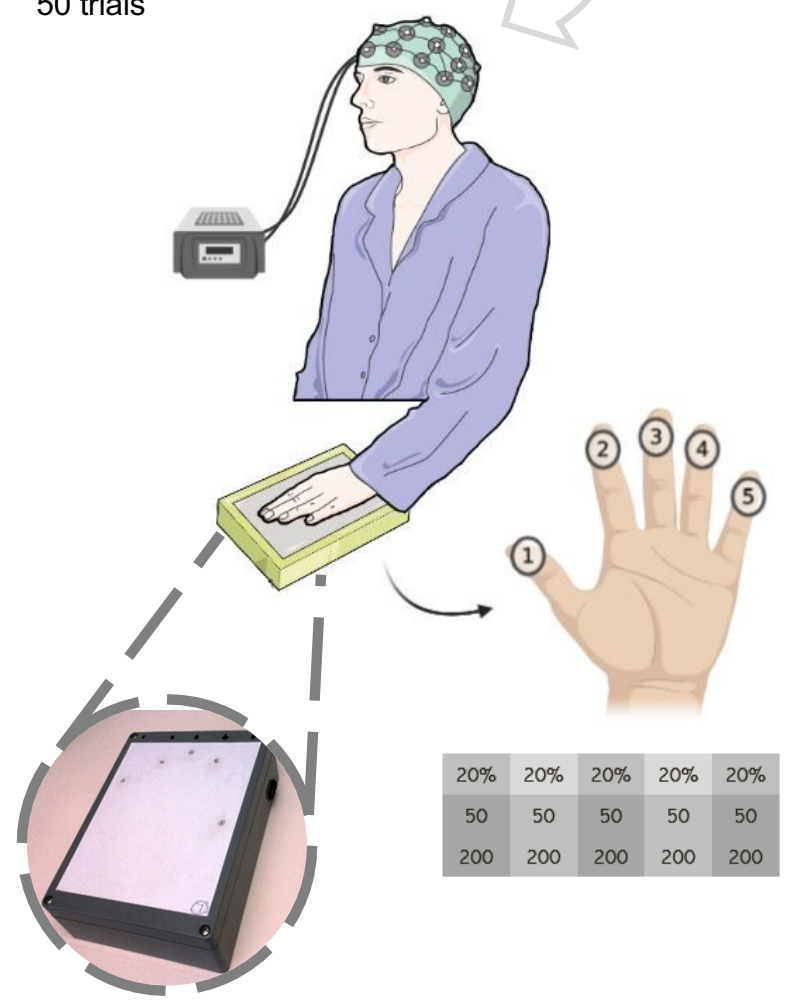

\section{B. ACTIVE CONDITION}

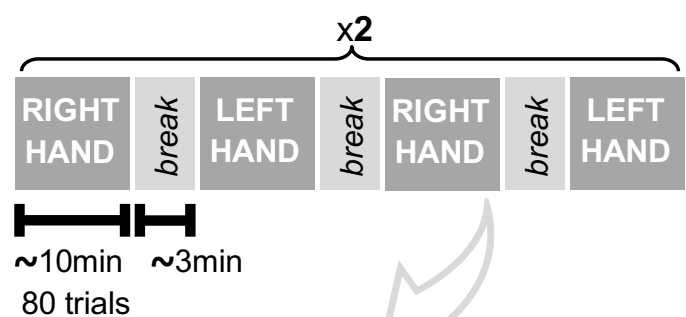

80 trials

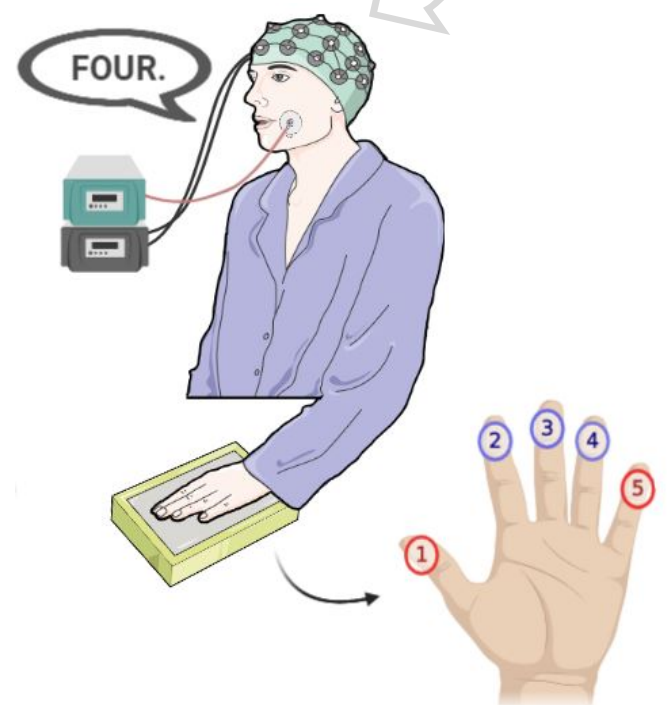

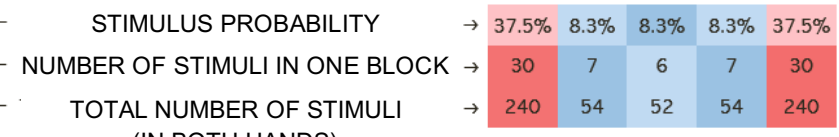

(IN BOTH HANDS)

During the study visit, participants gave written informed consent and were sitting comfortably as the EEG cap was placed and prepared for recording. The handboxes (in the picture above) were then calibrated to deliver soft touch stimuli with the same force to the fingertips of each digit and soft ear plugs were provided to reduce any external auditory input and help participants focusing on the tactile stimuli. (A) In the passive condition the order of digit stimulation was randomised within each block (on each hand in turn). Touches were delivered at a predefined frequency of $1 \mathrm{~Hz}$ (duration: 50ms; ISI: $950 \mathrm{~ms})$. Each of the 4 ( $\sim 5$ minutes-long) blocks contained 250 trials (50 stimulations per digit), for a total of 1000 trials (500 per hand and 100 per individual fingers) in $\sim 20$ minutes. A 10 seconds break after was offered after every block to improve participant's comfort. (B) In the active condition, digit stimulation was pseudo-randomised in each hand / block, with D1 
and D5 receiving 30 stimulations each (probability $=37.5 \%$ ), for a total of 240 touches each across the two hands (120 on the left and 120 on the right). The inner digits (D2, D3 and D4) shared the remaining 20 trials of each block (probability $=8.3 \%$ ), reaching $\sim 54$ stimulations each across the two hands ( 27 on each hand). Hence, of the total 640 active trials, 480 were dedicated to "expected" fingers (D1 and D5) and 160 to the "rare" central fingers (D2-D4). A microphone attached to the EMG recording was used to capture the participant's reaction time (resulting from the subtraction of the stimulus onset time from the start of the voice deflection on the EMG). The answer was recorded manually by the experimenter by typing the number on the computer and triggering the next stimulus. A maximum time lock was set on three seconds per trial. 
A.

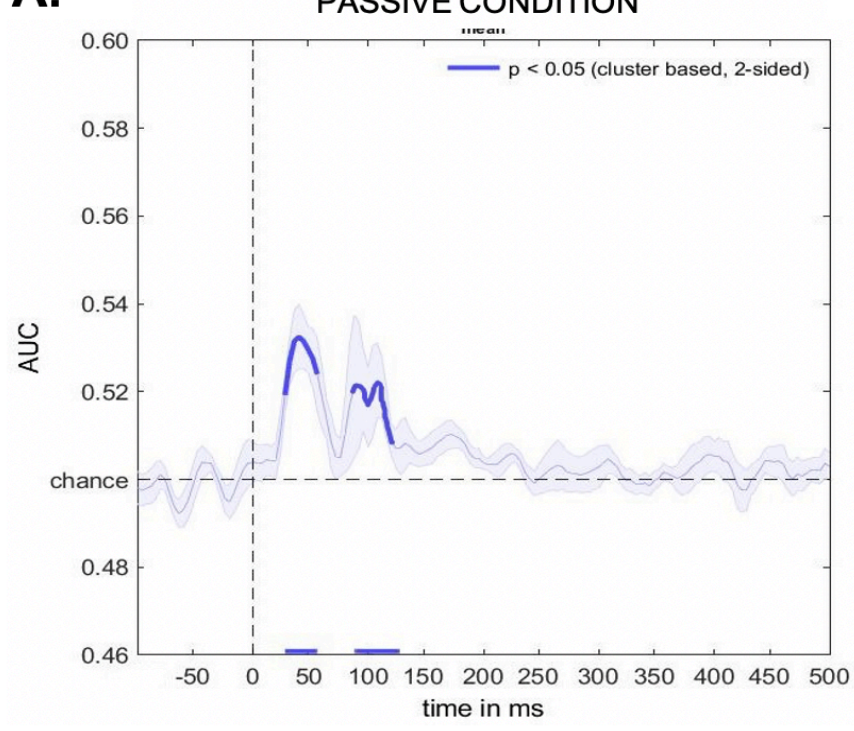

C.

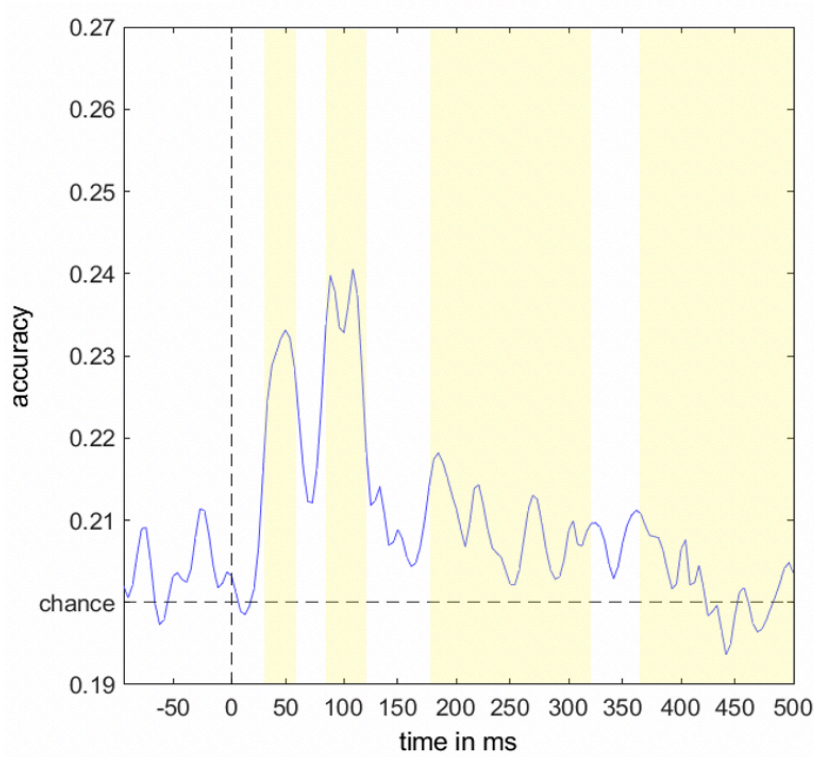

B.

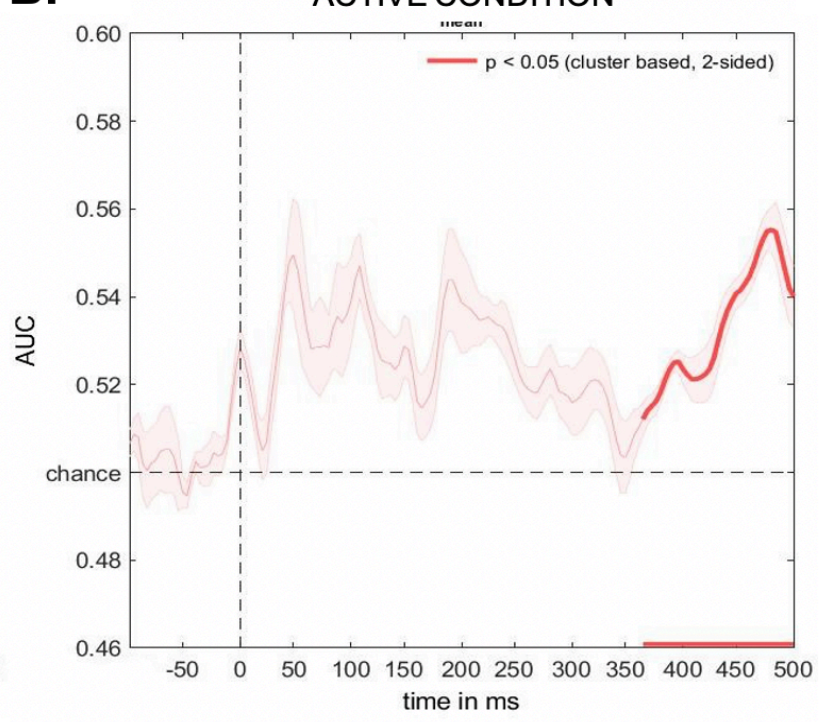

D.

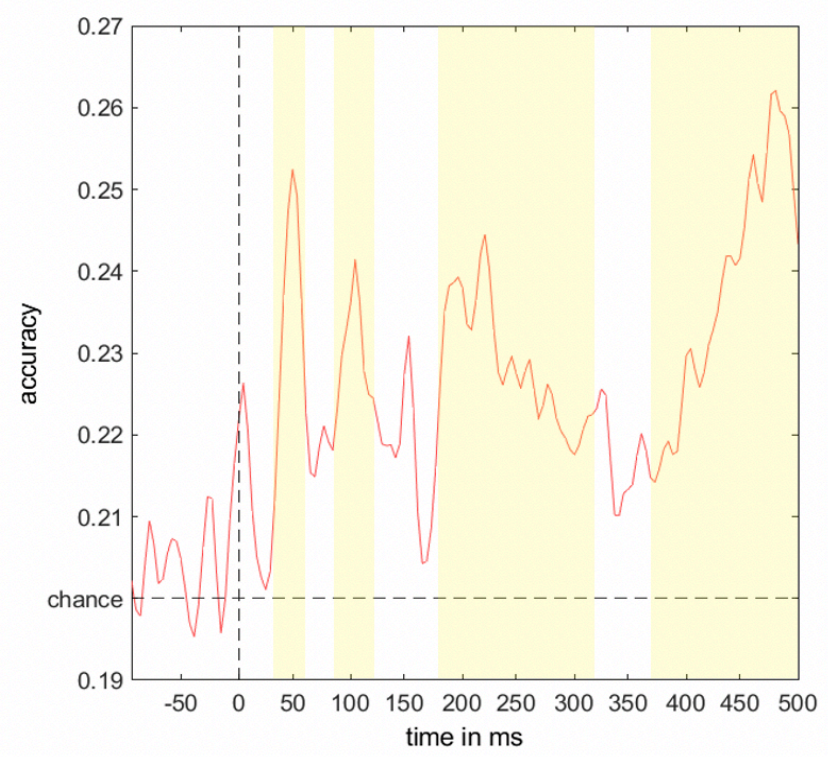

A, B. The average time-course of five one-vs-all classifiers trained and tested on the healthy "super-subject" data in the passive (A) and active (B) condition. The portion of time that survived cluster-based permutation test for significance against chance is highlighted with a thicker line. C, D. The decoding time-course of the all-vs-all classifier applied to the passive (C) and active (D) super-subject data, with the four time-windows selected for further testing highlighted in yellow. 
Figure 3 - GROUP-LEVEL DECODING OUTPUT

A.

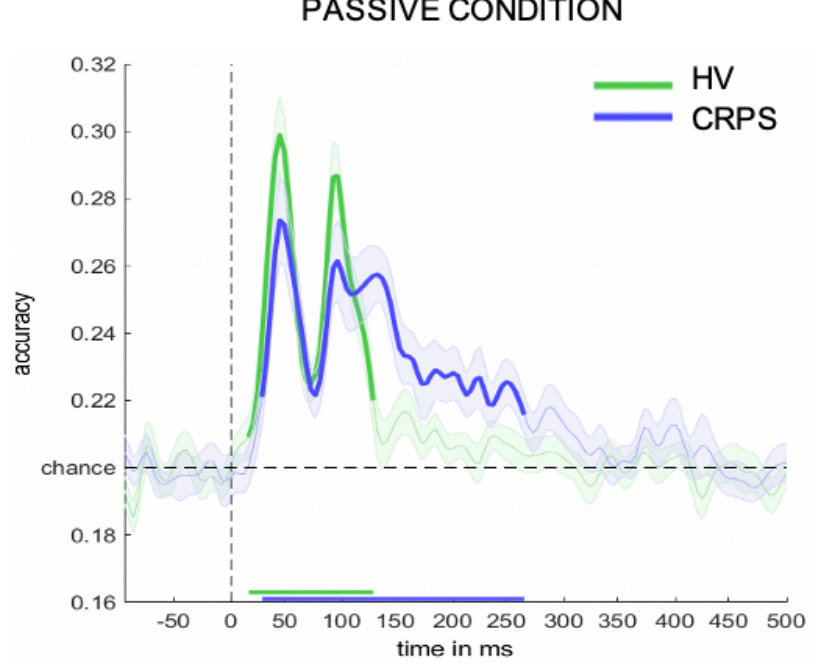

c.

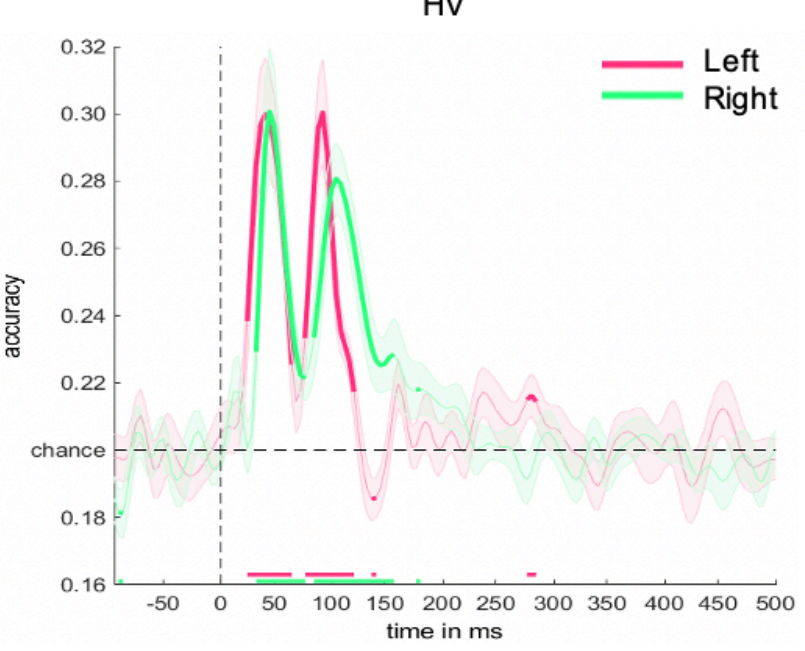

E.

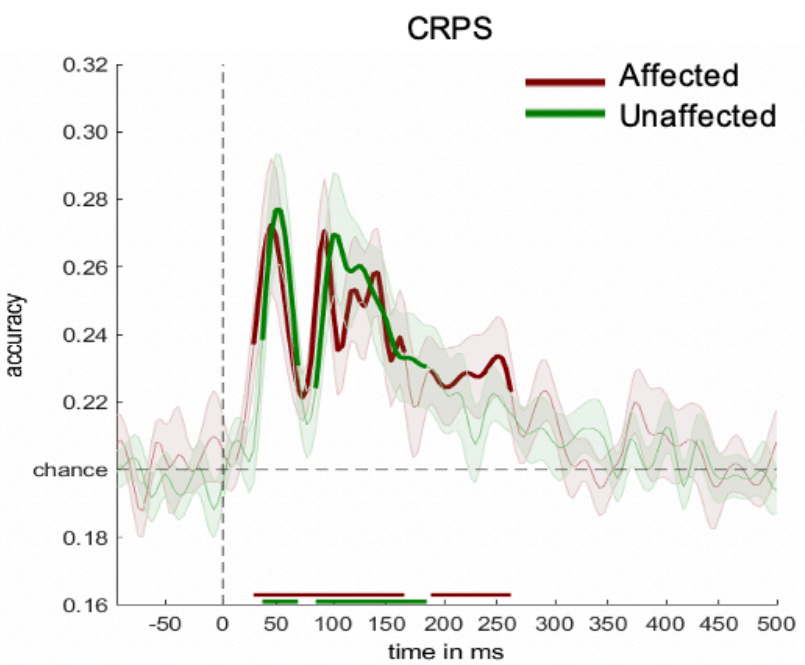

B.

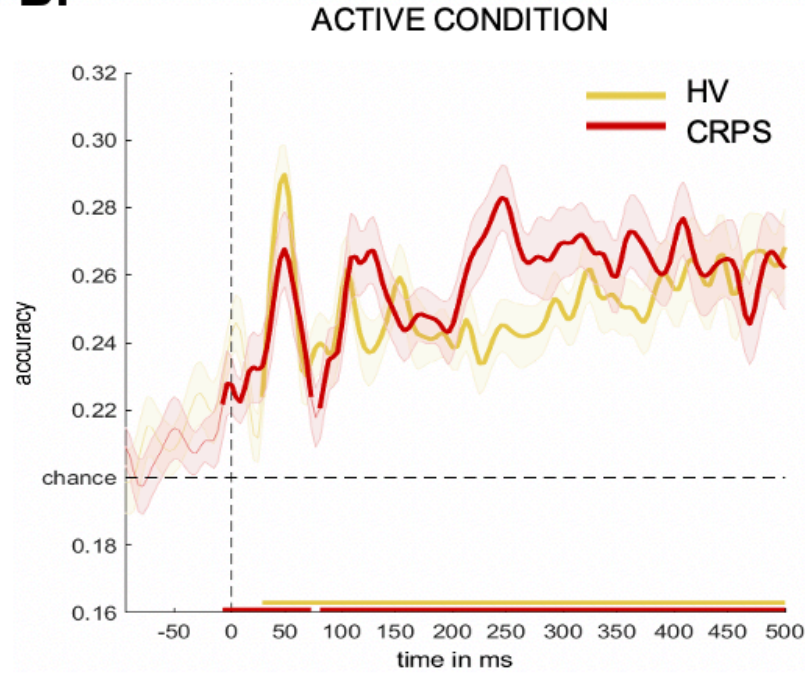

D.

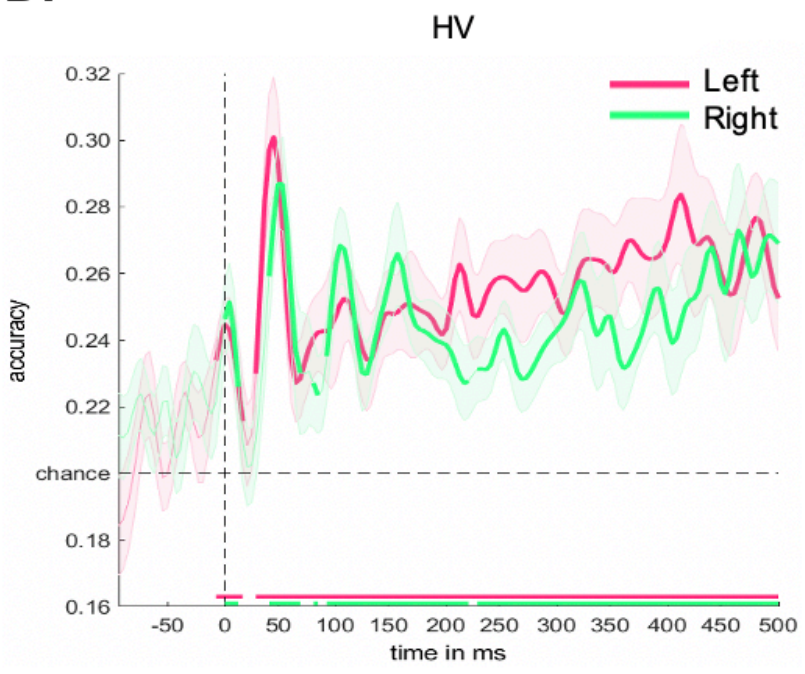

F.

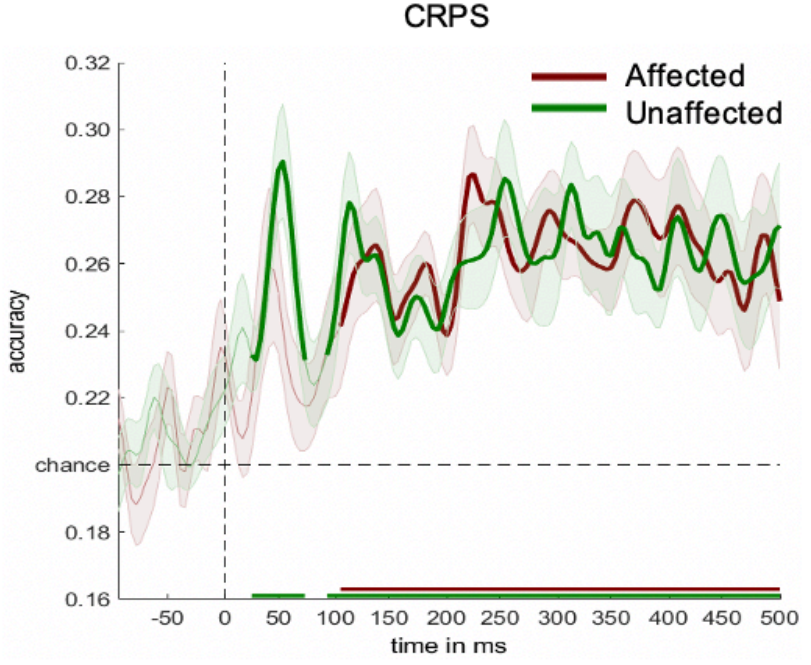


(A) Decoding performance (CA) as a function of time, resulting from training and testing two all-vs-all classifiers on the passive condition data from healthy volunteers (in green) and CRPS patients (in blue). (B) the time-course of the same two all-vs-all classifiers trained on the active condition, for healthy controls (in yellow) and CRPS patients (in red). (C) the passive and (D) active time-courses of classification performance on the left (in pink) and on the right hand (in light green) in the HV group only. (E) the passive and (F) active decoding performance time-course on the affected (in red) and on the unaffected side (in dark green) of the CRPS group only. In all of the above plots, the portion of time where classifier performed significantly better chance $(\mathrm{RCR}=20 \%)$ was established by means of t-test (i.e., uncorrected p-values $>0.05)$ is highlighted with a thicker line. The output of MVPA applied to successive time points, as pictured in the six graphs, is meant to describe when and for how long the information of interest, i.e., the identity of the finger that is being touched, becomes "encoded" or "represented" explicitly in the brain (Grootswagers et al., 2017). 
A.

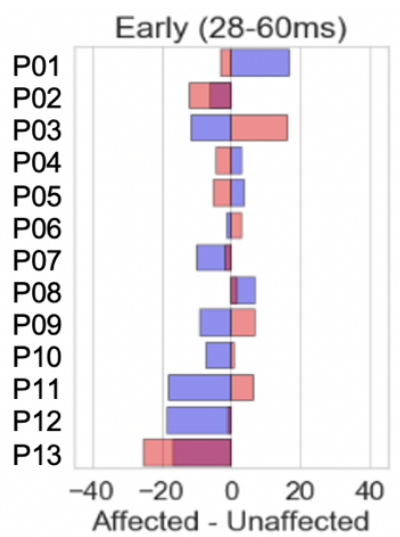

B.

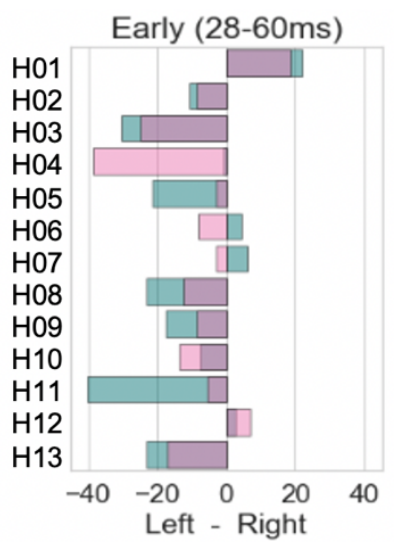

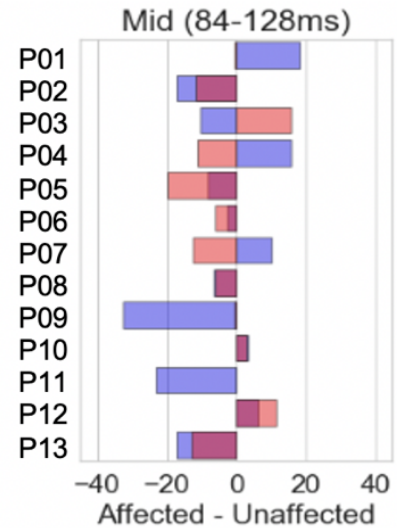
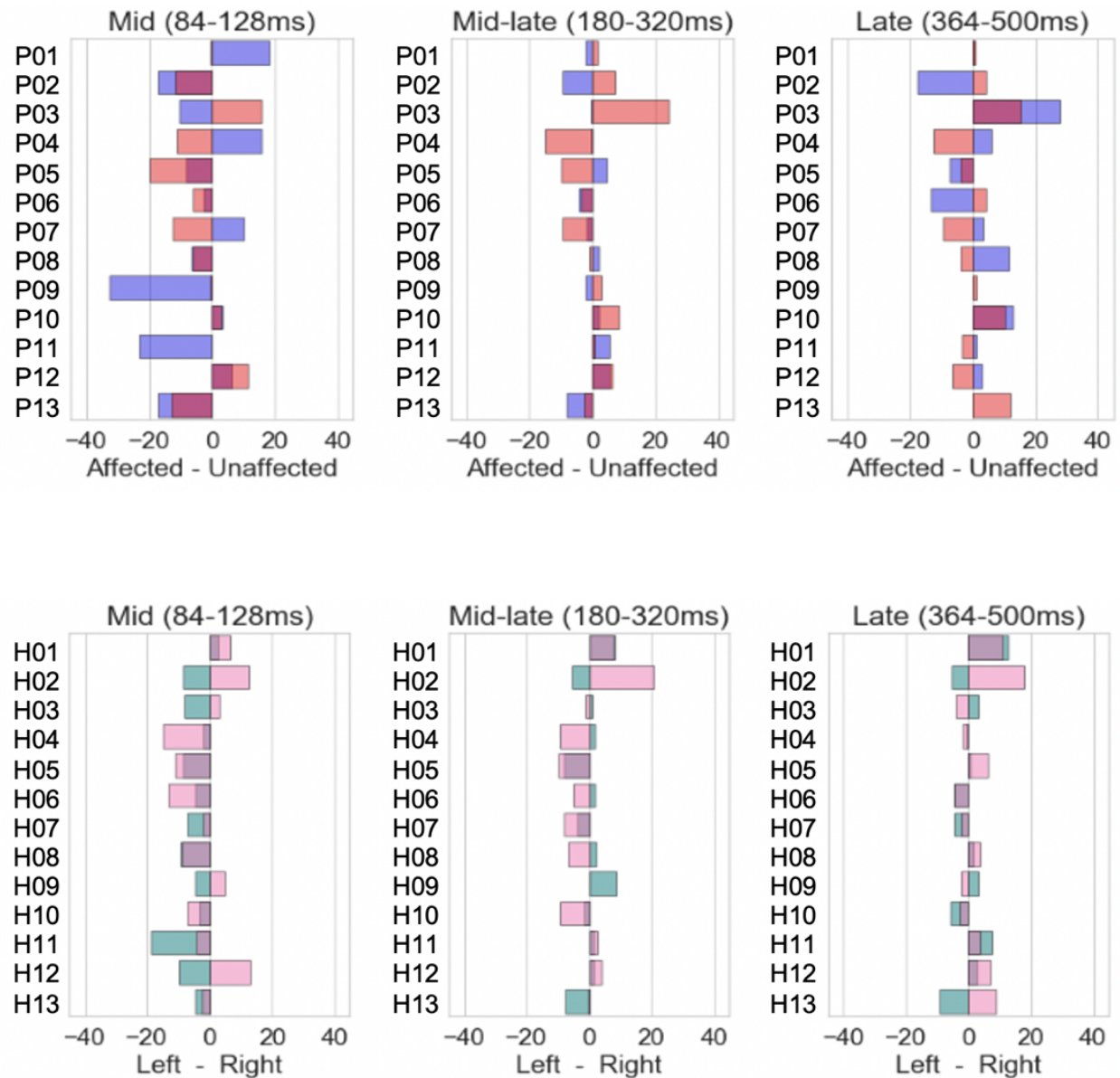

(A) Variability within the CRPS group. The four bar plots represent the subject-wise differences in mean decoding performance score between affected and unaffected side of the body (affected - unaffected), or "decodability imbalance", across the early, mid, mid-late and late time-window. For each patient, two decodability imbalances are drawn for the passive (in blue) and for the active condition (in red). When these two bars overlap, the decodability imbalance will be similar (in direction and amplitude) among the two conditions. Patients that had higher decoding performance on the unaffected compared to the affected side (following our expectations) will show negative decodability imbalance values (on the left side of each graph: affected $<$ unaffected), whereas those for whom the opposite was true (accuracy in the affected side was higher than the healthy side) will report positive decodability imbalances, hence leaning towards the right of each graph (affected $>$ unaffected). (B) Decodability imbalances between left and right hand of HVs were also reported for comparison. Here, imbalances in the passive condition are represented in cyan, whereas the active condition is reported in pink. Analogously, volunteers who showed higher decoding performance on the right 
compared to the left side (as expected) will have negative decodability imbalance values (on the left side of each graph), whereas left $>$ right outcomes result in positive decodability differences (on the right of each graph). 

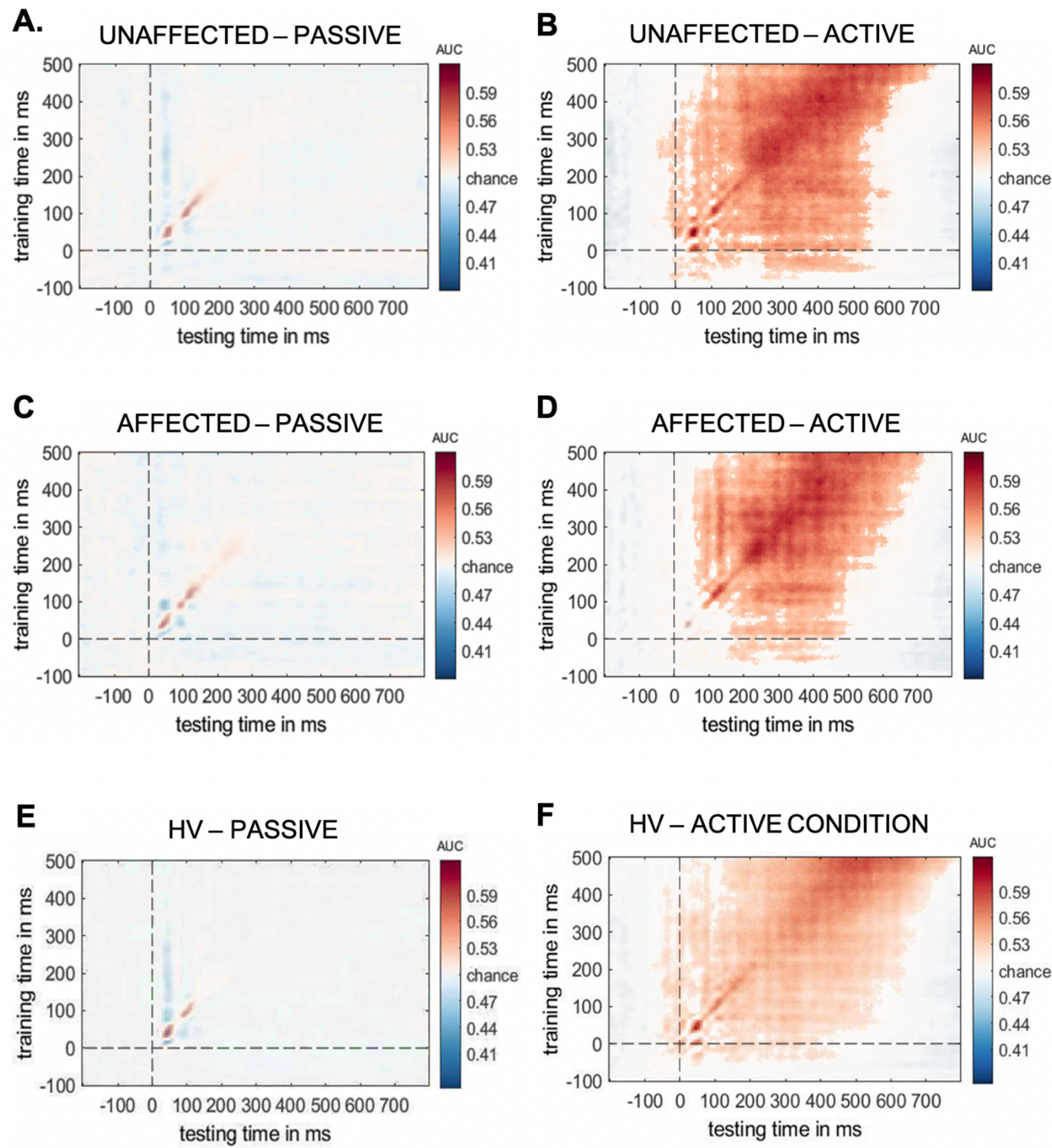

Temporal generalization matrices representing the generalization across time for (A) CRPS patients' unaffected side of body in the passive condition, and (B) their unaffected side data in the active condition; (C) CRPS patients' side affected by the disease in the passive condition, and (D) their affected side data during the active condition. Healthy volunteers' temporal generalizations are also plotted for comparison in the (E) passive condition, and (F) in the active condition. In each of the six graphs, training timepoints are represented on the y-axis, while testing timepoints are displayed on the $\mathrm{x}$-axis and two dashed lines locate the time of 
tactile stimulus onset in both dimensions. Color indicates decoder's performance strength (i.e., classification accuracy). The diagonal values (from the left bottom to top right) closely correspond to the outcome computed in the previous analysis (represented in Figure 3E, 3F and 3A, 3B). Higher off-diagonal performance indicates stronger temporal generalization (i.e., stability of neural representations). 
A.

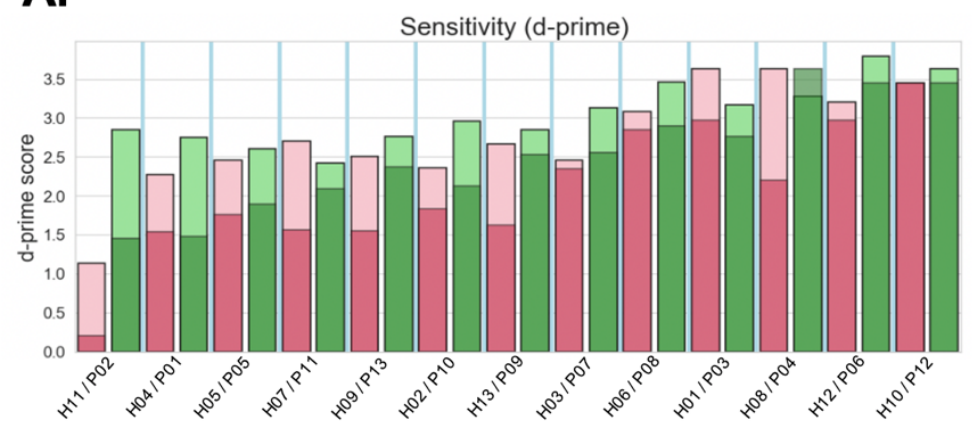

B.

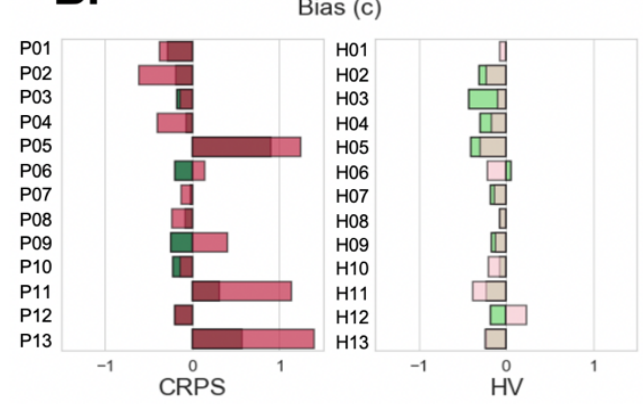

HV - left side $\quad$ HV - right side $\quad$ CRPS - affected side

CRPS - unaffected side
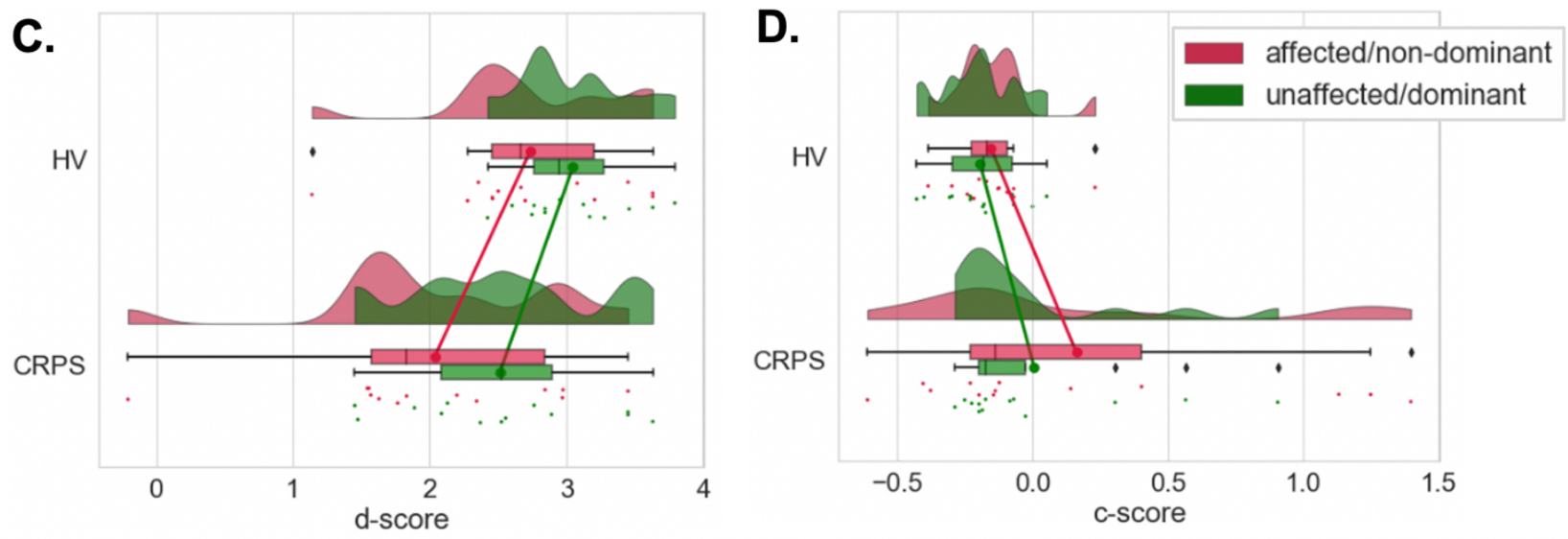

(A, B) Bar plots representing the subject-wise (A) d' score and (B) bias scores calculated between digits 1-5 ("expected fingers") and 2-3-4 ("rare fingers"). The affected / non-dominant side is represented in red / pink (for CRPS and HV respectively), and in the unaffected / dominant side is represented in dark / light green (for CRPS and HV respectively). The bars representing CRPS patients' values are drawn on top of the HV values, ordered from lowest to highest value. (C) distribution of d' scores in the two sides of HV and CRPS (above and below respectively). (D) distribution of c (bias) scores in the two groups and hands. The same color scheme was adopted (red for affected / non-dominant and green for unaffected / dominant) and the two transversal lines provide a representation of the side-specific mean difference between groups. 


\section{References}

(2013) World Medical Association Declaration of Helsinki: ethical principles for medical research involving human subjects. Jama, 310, 2191-2194.

Apkarian, A.V., Sosa, Y., Krauss, B.R., Thomas, P.S., Fredrickson, B.E., Levy, R.E., Harden, R.N. \& Chialvo, D.R. (2004) Chronic pain patients are impaired on an emotional decision-making task. Pain, 108, 129-136.

Bang, J.W. \& Rahnev, D. (2017) Stimulus expectation alters decision criterion but not sensory signal in perceptual decision making. Scientific Reports, 7.

Bantick, S.J., Wise, R.G., Ploghaus, A., Clare, S., Smith, S.M. \& Tracey, I. (2002) Imaging how attention modulates pain in humans using functional MRI. Brain, 125, 310-319.

Birklein, F., Ajit, S.K., Goebel, A., Perez, R. \& Sommer, C. (2018) Complex regional pain syndrome phenotypic characteristics and potential biomarkers. Nat Rev Neurol, 14, 272-284.

Blanke, O., Slater, M. \& Serino, A. (2015) Behavioral, Neural, and Computational Principles of Bodily SelfConsciousness. Neuron, 88, 145-166.

Borchers, A.T. \& Gershwin, M.E. (2014) Complex regional pain syndrome: a comprehensive and critical review. Autoimmunity reviews, 13, 242-265.

Bray, H. \& Moseley, G.L. (2011) Disrupted working body schema of the trunk in people with back pain. British Journal of Sports Medicine, 45, 168.

Brown, C.A., Scholtes, I., Shenker, N. \& Lee, M.C. (2020) Suboptimal learning of tactile-spatial predictions in patients with complex regional pain syndrome. Pain, 161, 369-378.

Bruehl, S. (2015) Complex regional pain syndrome. BMJ (Clinical research ed.), 351, h2730.

Bultitude, J.H., Walker, I. \& Spence, C. (2017) Space-based bias of covert visual attention in complex regional pain syndrome. Brain, 140, 2306-2321.

Chan, S.C.C., Chan, C.C.H., Kwan, A.S.K., Ting, K.-h. \& Chui, T.-y. (2012) Orienting attention modulates pain perception: an ERP study. PLoS One, 7, e40215-e40215.

Chennu, S., Noreika, V., Gueorguiev, D., Blenkmann, A., Kochen, S., Ibanez, A., Owen, A.M. \& Bekinschtein, T.A. (2013) Expectation and attention in hierarchical auditory prediction. $J$ Neurosci, 33, 11194-11205.

Clauwaert, A., Torta, D.M., Forster, B., Danneels, L. \& Van Damme, S. (2020) Somatosensory attentional modulations during pain-related movement execution. Experimental brain research, 238, 11691176. 
Delorme, A. \& Makeig, S. (2004) EEGLAB: an open source toolbox for analysis of single-trial EEG dynamics including independent component analysis. Journal of neuroscience methods, 134, 9-21.

Di Pietro, F., McAuley, J.H., Parkitny, L., Lotze, M., Wand, B.M., Moseley, G.L. \& Stanton, T.R. (2013) Primary somatosensory cortex function in complex regional pain syndrome: a systematic review and meta-analysis. The journal of pain : official journal of the American Pain Society, 14, 1001-1018.

Di Pietro, F., Stanton, T.R., Moseley, G.L., Lotze, M. \& McAuley, J.H. (2015) Interhemispheric somatosensory differences in chronic pain reflect abnormality of the healthy side. Human brain mapping, 36, 508-518.

Dick, B.D., Connolly, J.F., McGrath, P.J., Finley, G.A., Stroink, G., Houlihan, M.E. \& Clark, A.J. (2003) The disruptive effect of chronic pain on mismatch negativity. Clin Neurophysiol, 114, 1497-1506.

Ehrsson, H.H., Spence, C. \& Passingham, R.E. (2004) That\&\#039;s My Hand! Activity in Premotor Cortex Reflects Feeling of Ownership of a Limb. Science, 305, 875.

Fahrenfort, J.J., van Driel, J., van Gaal, S. \& Olivers, C.N.L. (2018) From ERPs to MVPA Using the Amsterdam Decoding and Modeling Toolbox (ADAM). Front Neurosci, 12, 368.

Feldman, H. \& Friston, K.J. (2010) Attention, uncertainty, and free-energy. Frontiers in Human Neuroscience, 4.

Feliu, M.H. \& Edwards, C.L. (2010) Psychologic factors in the development of complex regional pain syndrome: history, myth, and evidence. Clin J Pain, 26, 258-263.

Flor, H., Braun, C., Elbert, T. \& Birbaumer, N. (1997) Extensive reorganization of primary somatosensory cortex in chronic back pain patients. Neuroscience Letters, 224, 5-8.

Flor, H., Elbert, T., Knecht, S., Wienbruch, C., Pantev, C., Birbaumers, N., Larbig, W. \& Taub, E. (1995) Phantom-limb pain as a perceptual correlate of cortical reorganization following arm amputation. Nature, 375, 482-484.

Förderreuther, S., Sailer, U. \& Straube, A. (2004) Impaired self-perception of the hand in complex regional pain syndrome (CRPS). Pain, 110, 756-761.

Franz, M., Nickel, M.M., Ritter, A., Miltner, W.H. \& Weiss, T. (2015) Somatosensory spatial attention modulates amplitudes, latencies, and latency jitter of laser-evoked brain potentials. Journal of neurophysiology, 113, 2760-2768.

Friston, K. (2018) Does predictive coding have a future? Nature Neuroscience, 21, 1019-1021.

Garrido, M.I., Kilner, J.M., Stephan, K.E. \& Friston, K.J. (2009) The mismatch negativity: a review of underlying mechanisms. Clin Neurophysiol, 120, 453-463. 
Geha, P.Y., Baliki, M.N., Harden, R.N., Bauer, W.R., Parrish, T.B. \& Apkarian, A.V. (2008) The Brain in Chronic CRPS Pain: Abnormal Gray-White Matter Interactions in Emotional and Autonomic Regions. Neuron, 60, 570-581.

Grootswagers, T., Wardle, S.G. \& Carlson, T.A. (2017) Decoding Dynamic Brain Patterns from Evoked Responses: A Tutorial on Multivariate Pattern Analysis Applied to Time Series Neuroimaging Data. J Cogn Neurosci, 29, 677-697.

Halicka, M., Vitterso, A.D., Proulx, M.J. \& Bultitude, J.H. (2020) Neuropsychological Changes in Complex Regional Pain Syndrome (CRPS). Behav Neurol, 2020, 4561831.

Harden, R.N., Bruehl, S., Stanton-Hicks, M. \& Wilson, P.R. (2007) Proposed new diagnostic criteria for complex regional pain syndrome. Pain Med, 8, 326-331.

King, J.R. \& Dehaene, S. (2014) Characterizing the dynamics of mental representations: the temporal generalization method. Trends Cogn Sci, 18, 203-210.

Kuttikat, A., Noreika, V., Chennu, S., Shenker, N., Bekinschtein, T. \& Brown, C.A. (2018) Altered Neurocognitive Processing of Tactile Stimuli in Patients with Complex Regional Pain Syndrome. The journal of pain : official journal of the American Pain Society, 19, 395-409.

Kuttikat, A., Noreika, V., Shenker, N., Chennu, S., Bekinschtein, T. \& Brown, C.A. (2016) Neurocognitive and Neuroplastic Mechanisms of Novel Clinical Signs in CRPS. Front Hum Neurosci, 10, 16.

Kuttikat, A., Shaikh, M., Oomatia, A., Parker, R. \& Shenker, N. (2017) Novel Signs and Their Clinical Utility in Diagnosing Complex Regional Pain Syndrome (CRPS): A Prospective Observational Cohort Study. Clin J Pain, 33, 496-502.

Lancaster, J., Mano, H., Callan, D., Kawato, M. \& Seymour, B. (Year) Decoding acute pain with combined EEG and physiological data. 2017 8th International IEEE/EMBS Conference on Neural Engineering (NER). City. p. 521-524.

Lee, D.-H., Lee, K.-J., Cho, K.I.K., Noh, E.C., Jang, J.H., Kim, Y.C. \& Kang, D.-H. (2015) Brain Alterations and Neurocognitive Dysfunction in Patients With Complex Regional Pain Syndrome. The Journal of Pain, 16, 580-586.

Lenggenhager, B., Tadi, T., Metzinger, T. \& Blanke, O. (2007) Video Ergo Sum: Manipulating Bodily SelfConsciousness. Science, 317, 1096.

Lenz, M., Höffken, O., Stude, P., Lissek, S., Schwenkreis, P., Reinersmann, A., Frettlöh, J., Richter, H., Tegenthoff, M. \& Maier, C. (2011) Bilateral somatosensory cortex disinhibition in complex regional pain syndrome type I. Neurology, 77, 1096-1101.

Lewis, J.S. \& Schweinhardt, P. (2012) Perceptions of the painful body: the relationship between body perception disturbance, pain and tactile discrimination in complex regional pain syndrome. European journal of pain (London, England), 16, 1320-1330.

Luck, S. (2005) Ten simple rules for designing ERP Experiments, pp. 17-32. 
Lueders, H., Lesser, R.P., Hahn, J., Dinner, D.S. \& Klem, G. (1983) Cortical somatosensory evoked potentials in response to hand stimulation. Journal of neurosurgery, 58, 885-894.

Macmillan, N. \& Creelman, D. (2004) Detection Theory: A User's Guide.

Maihöfner, C., Handwerker, H.O., Neundörfer, B. \& Birklein, F. (2003) Patterns of cortical reorganization in complex regional pain syndrome. Neurology, 61, 1707-1715.

Maihöfner, C., Handwerker, H.O., Neundörfer, B. \& Birklein, F. (2004) Cortical reorganization during recovery from complex regional pain syndrome. Neurology, 63, 693.

Mancini, F., Wang, A.P., Schira, M.M., Isherwood, Z.J., McAuley, J.H., Iannetti, G.D., Sereno, M.I., Moseley, G.L. \& Rae, C.D. (2019) Fine-Grained Mapping of Cortical Somatotopies in Chronic Complex Regional Pain Syndrome. J Neurosci, 39, 9185-9196.

Marinus, J., Moseley, G.L., Birklein, F., Baron, R., Maihofner, C., Kingery, W.S. \& van Hilten, J.J. (2011) Clinical features and pathophysiology of complex regional pain syndrome. Lancet Neurol, 10, 637648.

Mima, T., Nagamine, T., Nakamura, K. \& Shibasaki, H. (1998) Attention Modulates Both Primary and Second Somatosensory Cortical Activities in Humans: A Magnetoencephalographic Study. Journal of Neurophysiology, 80, 2215-2221.

Moran, R.J., Campo, P., Symmonds, M., Stephan, K.E., Dolan, R.J. \& Friston, K.J. (2013) Free energy, precision and learning: the role of cholinergic neuromodulation. J Neurosci, 33, 8227-8236.

Mouraux, A. \& Iannetti, G.D. (2018) The search for pain biomarkers in the human brain. Brain, 141, 32903307.

Pfannmöller, J., Strauss, S., Langner, I., Usichenko, T. \& Lotze, M. (2019) Investigations on maladaptive plasticity in the sensorimotor cortex of unilateral upper limb CRPS I patients. Restorative neurology and neuroscience, 37, 143-153.

Pleger, B., Tegenthoff, M., Schwenkreis, P., Janssen, F., Ragert, P., Dinse, H.R., Völker, B., Zenz, M. \& Maier, C. (2004) Mean sustained pain levels are linked to hemispherical side-to-side differences of primary somatosensory cortex in the complex regional pain syndrome I. Experimental brain research, 155, 115-119.

Polich, J. (2007) Updating P300: an integrative theory of P3a and P3b. Clin Neurophysiol, 118, 2128-2148.

Popkirov, S., Hoeritzauer, I., Colvin, L., Carson, A.J. \& Stone, J. (2019) Complex regional pain syndrome and functional neurological disorders - time for reconciliation. Journal of neurology, neurosurgery, and psychiatry, 90, 608-614.

Rosa, M.J. \& Seymour, B. (2014) Decoding the matrix: Benefits and limitations of applying machine learning algorithms to pain neuroimaging. PAIN®, 155, 864-867. 
Şahin, M. \& Aybek, E. (2019) Jamovi: An Easy to Use Statistical Software for the Social Scientists. International Journal of Assessment Tools in Education, 6, 670-692.

Schubert, R., Blankenburg, F., Lemm, S., Villringer, A. \& Curio, G. (2006) Now you feel it—now you don't: ERP correlates of somatosensory awareness. Psychophysiology, 43, 31-40.

Schubert, R., Ritter, P., Wüstenberg, T., Preuschhof, C., Curio, G., Sommer, W. \& Villringer, A. (2008) Spatial Attention Related SEP Amplitude Modulations Covary with BOLD Signal in S1-A Simultaneous EEG-fMRI Study. Cerebral Cortex, 18, 2686-2700.

Schulz, E., Zherdin, A., Tiemann, L., Plant, C. \& Ploner, M. (2011) Decoding an Individual's Sensitivity to Pain from the Multivariate Analysis of EEG Data. Cerebral Cortex, 22, 1118-1123.

Swart, C.M., Stins, J.F. \& Beek, P.J. (2009) Cortical changes in complex regional pain syndrome (CRPS). European journal of pain (London, England), 13, 902-907.

Tu, Y., Hung, Y.S., Zhang, Z. \& Hu, L. (Year) Prediction of pain perception using multivariate pattern analysis of laser-evoked EEG oscillations. 2014 13th International Conference on Control Automation Robotics \& Vision (ICARCV). City. p. 13-16.

van Velzen, G.A., Rombouts, S.A., van Buchem, M.A., Marinus, J. \& van Hilten, J.J. (2016) Is the brain of complex regional pain syndrome patients truly different? European journal of pain (London, England), 20, 1622-1633.

Walton, K.D., Dubois, M. \& Llinás, R.R. (2010) Abnormal thalamocortical activity in patients with Complex Regional Pain Syndrome (CRPS) type I. Pain, 150, 41-51.

Wickens, T.D. (2002) Elementary signal detection theory. Oxford University Press, USA.

Wittayer, M., Dimova, V., Birklein, F. \& Schlereth, T. (2018) Correlates and importance of neglect-like symptoms in complex regional pain syndrome. Pain, 159, 978-986.

Woo, C.W., Schmidt, L., Krishnan, A., Jepma, M., Roy, M., Lindquist, M.A., Atlas, L.Y. \& Wager, T.D. (2017) Quantifying cerebral contributions to pain beyond nociception. Nat Commun, 8, 14211.

Wood, C.C., Spencer, D.D., Allison, T., McCarthy, G., Williamson, P.D. \& Goff, W.R. (1988) Localization of human sensorimotor cortex during surgery by cortical surface recording of somatosensory evoked potentials. Journal of neurosurgery, 68, 99-111.

Yvon, A., Faroni, A., Reid, A.J. \& Lees, V.C. (2018) Selective Fiber Degeneration in the Peripheral Nerve of a Patient With Severe Complex Regional Pain Syndrome. Front Neurosci, 12, 207. 